\title{
Black hole growth through hierarchical black hole mergers in dense star clusters: implications for gravitational wave detections
}

\author{
Fabio Antonini, ${ }^{1 \star}$ Mark Gieles, ${ }^{1,2,3}$ and Alessia Gualandris, ${ }^{1}$ \\ ${ }^{1}$ Faculty of Engineering and Physical Sciences, University of Surrey, Guildford, Surrey, GU2 7XH, United Kingdom \\ ${ }^{2}$ Institut de Ciències del Cosmos (ICCUB), Universitat de Barcelona, Martí i Franquès 1, E08028 Barcelona, Spain \\ ${ }^{3}$ ICREA, Pg. Lluis Companys 23, 08010 Barcelona, Spain.
}

Accepted XXX. Received YYY; in original form ZZZ

\begin{abstract}
In a star cluster with a sufficiently large escape velocity, black holes (BHs) that are produced by $\mathrm{BH}$ mergers can be retained, dynamically form new $\mathrm{BH}$ binaries, and merge again. This process can repeat several times and lead to significant mass growth. In this paper, we calculate the mass of the largest $\mathrm{BH}$ that can form through repeated $\mathrm{BH}$ mergers and determine how its value depends on the physical properties of the host cluster. We adopt an analytical model in which the energy generated by the black hole binaries in the cluster core is assumed to be regulated by the process of two-body relaxation in the bulk of the system. This principle is used to compute the hardening rate of the binaries and to relate this to the time-dependent global properties of the parent cluster. We demonstrate that in clusters with initial escape velocity $\gtrsim 300 \mathrm{~km} \mathrm{~s}^{-1}$ in the core and density $\gtrsim 10^{5} M_{\odot} \mathrm{pc}^{-3}$, repeated mergers lead to the formation of BHs in the mass range $100-10^{5} M_{\odot}$, populating any upper mass gap created by pair-instability supernovae. This result is independent of cluster metallicity and the initial $\mathrm{BH}$ spin distribution. We show that about $10 \%$ of the present-day nuclear star clusters meet these extreme conditions, and estimate that BH binary mergers with total mass $\gtrsim 100 M_{\odot}$ should be produced in these systems at a maximum rate $\approx 0.05 \mathrm{Gpc}^{-3} \mathrm{yr}^{-1}$, corresponding to one detectable event every few years with Advanced LIGO/Virgo at design sensitivity.
\end{abstract}

Key words: black hole physics - gravitational waves - stars: kinematics and dynamics

\section{INTRODUCTION}

Dynamical three-body interactions in the dense core of a star cluster lead to the efficient formation, hardening and merger of black hole (BH) binaries (Kulkarni et al. 1993; Sigurdsson \& Hernquist 1993). Numerical simulations based on Monte Carlo and $N$-body techniques (e.g., Portegies Zwart \& McMillan 1999; Downing et al. 2010; Banerjee et al. 2010; Tanikawa 2013; Rodriguez et al. 2015, 2016; Askar et al. 2017; Samsing et al. 2018) have shown that such dynamically formed binaries could explain many, or perhaps even most, of the $\mathrm{BH}$ mergers that have been observed by Advanced LIGO and Virgo (Abbott et al. 2016a, 2017c). However, it remains an open question what physical properties will allow to distinguish these systems from binaries which are produced in the field of a galaxy through either the evo-

^ f.antonini@surrey.ac.uk lution of isolated pairs of massive stars (Belczynski et al. 2002; Amaro-Seoane \& Chen 2016; Belczynski et al. 2016; Marchant et al. 2016; Mandel \& De Mink 2016; Gerosa et al. 2018; Spera et al. 2018; Amaro-Seoane 2018b) or in hierarchical triple systems via the Lidov-Kozai mechanism (Silsbee \& Tremaine 2017; Antonini et al. 2017, 2018; Rodriguez \& Antonini 2018; Liu \& Lai 2018). Currently, both cluster and field formation scenarios appear to be broadly consistent with the Advanced LIGO/Virgo detections (Abbott et al. 2016b).

A possible way to determine which formation mechanism is dominant is to compare the mass function of the detected binaries to model predictions. The BHs that are produced through three body dynamical processes in a dense star cluster might in fact be much heavier than those formed from the collapse of massive stars. Field formation scenarios predict long times between the formation and merger of a compact binary (e.g., Belczynski et al. 2002), so that repeated mergers should be nearly im- 
possible in this case. On the other hand, in star clusters with sufficiently large escape velocities, BHs that are produced by previous mergers can be retained after the momentum kick due to the merger, form new binaries, and merge again (Antonini \& Rasio 2016; Rodriguez et al. 2018). Statistically observable imprints in the properties of the newly formed BHs are expected (Antonini \& Rasio 2016; Gerosa \& Berti 2017; Fishbach et al. 2017; Rodriguez et al. 2018): they should be more massive than BHs born from "ordinary" stellar evolution; they should merge later, at lower redshift, than earlier generation BHs; and after a few mergers their dimensionless spin magnitudes (on average) should cluster around $\approx 0.7^{1}$ while if the holes have gained a significant fraction of their mass via minor mergers their spin should be low (e.g., Miller 2002).

During the inspiral of a binary $\mathrm{BH}$, gravitational wave (GW) radiation is emitted anisotropically due to asymmetries in the merger configuration. Numerical relativity simulations show that the kick velocity for non-spinning objects depends on the mass ratio of the binary $q=m_{2} / m_{1} \leq 1$ as $v_{\mathrm{GW}} \approx 175 \mathrm{~km} \mathrm{~s}^{-1} f(q)$, where $f(q)=68.5 \eta^{2}(1-4 \eta)^{1 / 2}(1-$ $0.93 \eta)$ and $\eta \equiv q /(1+q)^{2}$, which peaks at $f(q \approx 0.36)=1$ and vanishes at $f(0)=f(1)=0$. But, if the BHs have a finite spin, the kick could be much larger (Damour \& Gopakumar 2006; González et al. 2007b; Campanelli et al. 2007). Generally, whether significant $\mathrm{BH}$ growth occurs or not will depend on the host cluster physical properties. Rodriguez et al. (2018) set the initial spin of the BHs to zero and showed that in this case second-generation BHs can form in clusters with properties similar to current day globular clusters. However, the second generation BHs are promptly ejected after a second merger, so that no growth above about $100 M_{\odot}$ can ever occur in globular clusters.

In this paper, we compute the mass of the largest $\mathrm{BH}$ that can be formed through hierarchical mergers of smaller BHs, and determine how its value is set by the global properties (i.e., mass and averaged density) of the host cluster, including a prescription for the dynamical evolution of the cluster itself. The possibility of significant $\mathrm{BH}$ growth within a dense star cluster has been explored before (e.g., Quinlan \& Shapiro 1987; Davies et al. 2011; Lupi et al. 2014). Most of this previous work focused on the evolution of extremely dense clusters where a relativistic instability sets in, driving the formation of a massive seed via the "catastrophic" collapse of the cluster core. For this collapse to occur, however, the merger timescale of binaries due to the emission of gravitational radiation must be shorter than the timescale for cluster heating via binary-single encounters. This condition typically requires a cluster velocity dispersion $\gtrsim 1000 \mathrm{~km} \mathrm{~s}^{-1}$ (e.g., Lee 1995; Kupi et al. 2006), which is much larger than that measured in present-day star clusters.

Here, we work under the more conservative and realistic assumption that the dynamical hardening of $\mathrm{BH}$ binaries is an efficient energy source which complies with Hénon's principle (Hénon 1975). According to this principle, the rate of energy generation in the core is regulated by the energy

1 A dimensionless spin magnitude of one corresponds to a maximally spinning $\mathrm{BH}$, while zero spin magnitude corresponds to a non-spinning $\mathrm{BH}$. demands of the bulk of the system. Breen \& Heggie (2013) showed that a population of stellar-mass BHs can act as the central energy source, implying that there is thermal contact between the BHs and the stars. As a consequence, a BH population can be dynamically retained for as long as 10 halfmass relaxation timescales of the cluster. For the systems we consider here, the bulk of the system is in the light stellar component, while the energy flux must be supplied by the BHs, and ultimately must be generated by hard binaries in the core of the BH subsystem (e.g., Breen \& Heggie 2013). Hénon's principle allows us to relate the hardening rate of the binaries to the global properties of their parent cluster, as opposed to the core properties as done in the previous literature (e.g., Gültekin et al. 2004; Antonini \& Rasio 2016; D'Orazio \& Samsing 2018; Choksi et al. 2018). The advantage is that the energy generated by the binaries in the core can be easily linked to the secular evolution of the cluster, and thus a complete description of the cluster and binary evolution can be obtained.

In Section 2, we describe the analytical model that we used to compute the hardening rate of $\mathrm{BH}$ binaries and the evolution of the cluster in which they reside. We use this model to study the mass growth of a $\mathrm{BH}$ seed undergoing repeated mergers with smaller objects. In order to treat this problem analytically we first work under the simplifying assumption that dynamical and GW recoil kicks can be ignored. In Section 3, we add these and other additional ingredients to our models using a semi-analytical method. We adopt this method to follow the long term evolution of BHs in dense star clusters and to derive the properties of the merging binaries they produce. The astrophysical implications of our results are discussed in Section 4.

\section{BINARY HEATING}

Let us assume that the heating rate of $\mathrm{BH}$ binaries is so high that we need to invoke a "capped" heating rate, which is set by the maximum heat flow that can be conducted outward by two-body relaxation. This condition may not be met soon after the formation of the cluster, but because the dynamical friction time of $\mathrm{BHs}$ is short and $\mathrm{BH}$ binary formation is efficient, heating by BHs quickly goes up to generate the maximum heat flow. This means that the heat production in the centre by BHs is balanced by the global energy flow (Hénon 1961; Gieles et al. 2011; Breen \& Heggie 2013) and we can relate the heat generation to the cluster properties:

$\dot{E}=\zeta \frac{|E|}{\tau_{\mathrm{rh}}}$,

where $E \simeq-0.2 G M_{\mathrm{cl}}^{2} / r_{\mathrm{h}}$ is the total energy of the cluster, with $M_{\mathrm{cl}}$ the total cluster mass and $r_{\mathrm{h}}$ the half-mass radius. The constant $\zeta \simeq 0.1$ (Gieles et al. 2011; Alexander \& Gieles 2012 ), and $\tau_{\mathrm{rh}}$ is the average relaxation time-scale within $r_{\mathrm{h}}$ which is given by (e.g., Spitzer \& Hart 1971)

$\tau_{\mathrm{rh}}=0.138 \sqrt{\frac{M_{\mathrm{cl}} r_{\mathrm{h}}^{3}}{G}} \frac{1}{\left\langle m_{\star}\right\rangle \psi \ln \Lambda}$.

Here $\left\langle m_{\star}\right\rangle$ is the mean mass of the stars and stellar remnants and $\ln \Lambda$ is the Coulomb logarithm, which depends weakly on the total number of stars; we ignore this dependence and 
set $\ln \Lambda=10$. The constant $\psi$ depends on the mass spectrum within $r_{\mathrm{h}}$, and is often assumed to be $\psi=1$, which applies to equal-mass clusters. For a full mass spectrum, however, $\psi$ can be as high as $\psi \simeq 30-100$ (Gieles et al. 2010). In what follows, we adopt $\psi=5$. This takes into account that in the early evolution of the cluster (first $\sim 100 \mathrm{Myr}$ ) the mass function contains more massive stars and $\psi$ is high. After this time, as a result of the BHs, the mass function remains wide despite the turn-off mass being $\lesssim 1 M_{\odot}$. In the case of equipartition, Eq. (24) in Spitzer \& Hart (1971) states: $\psi=\left\langle m_{\star}^{2.5}\right\rangle /\left\langle m_{\star}\right\rangle^{2.5}$. If we approximate the cluster by two mass components, BHs with mass $10 M_{\odot}$ and stars with mass $0.6 M_{\odot}$, then for a mass fraction in $\mathrm{BHs}$ of a few percent we find $\psi \simeq 5$.

Combining the two expressions above we find for the heating rate for $\left\langle m_{\star}\right\rangle=0.6 M_{\odot}, \zeta=0.1$ and $\psi=5$

$\dot{E}_{0} \simeq 2.3 \times 10^{5} M_{\odot}\left(\mathrm{km} \mathrm{s}^{-1}\right)^{2} \mathrm{Myr}^{-1} M_{5}^{2 / 3} \rho_{5,0}^{5 / 6}$,

where the subscript 0 refers to initial values and we expressed the result in terms of $M_{5}=M_{\mathrm{cl}} / 10^{5} M_{\odot}$ and $\rho_{5,0}=$ $\rho_{\mathrm{h}, 0} / 10^{5} M_{\odot} / \mathrm{pc}^{3}$, with $\rho_{\mathrm{h}, 0}=3 M_{\mathrm{cl}} /\left(8 \pi r_{\mathrm{h}, 0}^{3}\right)$ the averaged density within the cluster half-mass radius.

In the absence of other effects, the cluster has a constant radius until $t=t_{0}$, where $t_{0}$ is the time for $\mathrm{BHs}$ to reach the centre and start heating the cluster. This is a fraction of the initial $\tau_{\text {rh }}$, i.e., $\lesssim 1$ Myr for the model parameters used about, hence we can safely assume that heating starts immediately and therefore $t_{0}=0$. In Section 3 we include larger $t_{0}$, because for the most massive clusters $t_{0}$ can be $\gtrsim 100 \mathrm{Myr}$. If we neglect mass loss from stellar evolution and the escape of BHs and stars (i.e., we assume a constant $M_{\mathrm{cl}}$ ), then the evolution of the cluster radius follows from the energy evolution and the assumption of virial equilibrium: $\dot{r}_{\mathrm{h}} / r_{\mathrm{h}}=\dot{E} /|E|$ and solving this gives (Hénon 1965)

$r_{\mathrm{h}}(t)=r_{\mathrm{h}, 0}\left(\frac{3}{2} \frac{\zeta t}{\tau_{\mathrm{rh}, 0}}+1\right)^{2 / 3}$,

such that

$v_{\mathrm{esc}}(t)=v_{\mathrm{esc}, 0}\left(\frac{3}{2} \frac{\zeta t}{\tau_{\mathrm{rh}, 0}}+1\right)^{-1 / 3}$,

where

$v_{\mathrm{esc}, 0} \simeq 50 \mathrm{~km} \mathrm{~s}^{-1} M_{5}^{1 / 3} \rho_{5,0}^{1 / 6}$,

$\tau_{\mathrm{rh}, 0} \simeq 7.5 \mathrm{Myr} M_{5} \rho_{5,0}^{-1 / 2}$.

The constant of proportionality in the escape velocity applies to a King (1966) model with $W_{0}=7$. Substituting these relations in Eq. (3) we find

$\dot{E}(t) \simeq \dot{E}_{0}\left(\frac{3}{2} \frac{\zeta t}{\tau_{\mathrm{rh}, 0}}+1\right)^{-5 / 3}$.

The rapid decrease of $\dot{E}$ with time is the result of cluster expansion, increasing $\tau_{\mathrm{rh}}$ and lowering $E$ (see Eq. 1).

Assuming that the heating is produced by the $\mathrm{BH}$ binaries in the core, it follows that the rate at which the core binaries harden is ${ }^{2}$

$\dot{E}_{\text {bin }}=-\dot{E}$,

${ }^{2}$ We note that in balanced evolution $\left|\dot{E}_{\text {bin }}\right|$ is actually slightly where $E_{\text {bin }}=-G m_{1} m_{2} / 2 a$, and $m_{1}$ and $m_{2}$ are the masses of the binary components. Here we have assumed that at any time one binary is responsible for most of the heat production in the cluster core.

We can compare our results to the scaling of the hardening rate often used in previous work (e.g. Heggie \& Hut 2003; Binney \& Tremaine 2011): $\dot{E}_{\text {bin }}=0.2 E_{\text {bin }} / \tau_{\text {enc }}$, where $\tau_{\text {enc }}^{-1} \simeq 8 \pi G \rho a / \sigma$ is the binary-single encounter timescale, and $\rho$ and $\sigma$ are the density and velocity dispersion of the BHs near the binary, respectively. A choice often made in the literature is to set $\rho$ equal to the density of stars in the core (Gültekin et al. 2004, 2006; Antonini \& Rasio 2016; Samsing \& D'Orazio 2018; Samsing 2017; Choksi et al. 2018). This choice, however, is quite arbitrary as the core density of the BHs is not known a priori and cannot be easily linked to the cluster global properties. For the King model used above, taking $m_{1}=$ $m_{2}=10 M_{\odot}$ one finds $\dot{E}_{\text {bin }} \simeq-\pi G^{2} m_{1} m_{2} \rho / \sigma=-7 \times$ $10^{2} M_{\odot}\left(\mathrm{km} \mathrm{s}^{-1}\right)^{2} \mathrm{Myr}^{-1} M_{\mathrm{cl}}^{-1 / 3} \rho_{\mathrm{h}}^{5 / 6}$. Our assumption of balanced equilibrium instead led to the different normalisation and scaling $\dot{E}_{\text {bin }} \simeq-2.3 \times 10^{5} M_{\odot}\left(\mathrm{km} \mathrm{s}^{-1}\right)^{2} \mathrm{Myr}^{-1} M_{\mathrm{cl}}^{2 / 3} \rho_{\mathrm{h}}^{5 / 6}$.

The much lower value for the binary heating rate used in some previous studies implies a non-equilibrium state where the rate of energy generation by the core binaries cannot sustain the cluster core against collapse. In this situation, the $\mathrm{BH}$ core will rapidly contract causing the binary-single interaction rate to increase until $\dot{E}_{\text {bin }}$ matches the value given by our Eq. (9), after which the balanced evolution will be restored. This fits in the view that the rate of flow of energy is controlled by the system as a whole, not by its core properties.

Eq. (9) can be equivalently expressed in terms of the binary semi-major axis,

$\dot{a}=\frac{2 a^{2}}{G m_{1} m_{2}} \dot{E}_{\text {bin }}$.

The lifetime of a BH binary is then

$\tau_{\text {bin }}=\int_{a_{\mathrm{m}}}^{a_{\mathrm{h}}} \frac{G m_{1} m_{2}}{2 a^{2}} \dot{E}_{\mathrm{bin}}^{-1} d a \simeq-\frac{G m_{1} m_{2}}{2 a_{\mathrm{m}}} \dot{E}_{\mathrm{bin}}^{-1}$,

with $a_{\mathrm{h}} \approx G \mu / \sigma^{2}$ the semi-major axis at the hard/soft boundary, where $\mu=m_{1} m_{2} /\left(m_{1}+m_{2}\right)$, and $a_{\mathrm{m}}$ the semi-major axis at which the sequence of hardening interactions terminates because either the binary merges or it is ejected from the cluster. This latter quantity is determined by

$a_{\mathrm{m}}=\max \left(a_{\mathrm{GW}}, a_{\mathrm{ej}}\right)$,

with $a_{\mathrm{GW}}$ and $a_{\mathrm{ej}}$ defined below. Note that when solving Eq. (11) we have taken $\dot{E}_{\text {bin }}$ out of the time integral, i.e., we have assumed that the cluster properties do not change over $\tau_{\text {bin. }}$. This is justified because $\left|E / E_{\text {bin }}\right| \sim M_{\mathrm{cl}} /\left(m_{1}+m_{2}\right) \gg 1$. Moreover, we have used the fact that $a_{\mathrm{m}} \ll a_{\mathrm{h}}$.

As the binary binding energy increases following a

larger than $\dot{E}$, because some of the binary energy is used to eject stars/BHs with velocities in excess of the local escape velocity. The removal of the binding energy of the star/BH contributes indirectly to the heating of the core, but the excess energy above the escape energy does not contribute to the heating. For singlemass clusters, $\left|\dot{E}_{\text {bin }}\right| \simeq 1.1 \dot{E}$ (Goodman 1984). 
binary-single interaction, from energy and momentum conservation one finds that the centre of mass receives a recoil kick with velocity (Miller \& Lauburg 2009)

$v_{2-1}^{2} \simeq 0.2 G \frac{m_{1} m_{2}}{m_{123}} \frac{q_{3}}{a}$,

where $q_{3}=m_{3} /\left(m_{1}+m_{2}\right)$, with $m_{3}$ the mass of the interloper, and $m_{123}=m_{1}+m_{2}+m_{3}$. In deriving Eq. (13) we have assumed that during each binary-single interaction the binding energy of the binary increases by a fixed fraction $\delta \approx 0.2$ (e.g., Quinlan 1996; Miller \& Hamilton 2002a). It follows that the binary is ejected from the cluster when its semi-major axis drops below (Antonini \& Rasio 2016):

$a_{\mathrm{ej}}=0.2 G \frac{m_{1} m_{2}}{m_{123}} \frac{q_{3}}{v_{\mathrm{esc}}^{2}} \simeq 0.1 \mathrm{AU}\left(\frac{m_{1} m_{2}}{m_{123}} q_{3} \frac{0.6}{M_{\odot}}\right)\left(\frac{50 \mathrm{~km} \mathrm{~s}^{-1}}{v_{\mathrm{esc}}}\right)^{2}$.

In reality, for the high velocity dispersion clusters considered here, most (if not all) binaries will merge before $a$ has decreased to $a_{\mathrm{ej}}$. Then, the semi-major axis at which a merger will occur is determined by requiring that the rate of energy loss due to dynamical hardening equals that due to GW radiation (Peters 1964):

$\left.\dot{a}\right|_{\mathrm{GW}}=-\frac{64}{5} \frac{G^{3} m_{1} m_{2}\left(m_{1}+m_{2}\right)}{c^{5} a^{3}\left(1-e^{2}\right)^{7 / 2}} g(e)$,

with $c$ the speed of light, $e$ the binary eccentricity, $g(e)=$ $\left(1+\frac{73}{24} e^{2}+\frac{37}{96} e^{4}\right)$, and the merger time is $\tau_{\mathrm{GW}}=a /|\dot{a}|_{\mathrm{GW}} \mid$. Solving $\left.\dot{a}\right|_{\mathrm{GW}}=\dot{a}$, gives

$$
\begin{array}{r}
a_{\mathrm{GW}}=\left(-\frac{32}{5} \frac{G^{4}\left(m_{1} m_{2}\right)^{2}\left(m_{1}+m_{2}\right) g(e)}{c^{5}\left(1-e^{2}\right)^{7 / 2}} \dot{E}_{\mathrm{bin}}^{-1}\right)^{1 / 5} \\
\simeq 10^{-2} \mathrm{AU}\left(\frac{\left(m_{1} m_{2}\right)^{2}\left(m_{1}+m_{2}\right)}{M_{\odot}^{5}} \frac{M_{\odot}\left(\mathrm{km} \mathrm{s}^{-1}\right)^{2} \mathrm{Myr}^{-1}}{\dot{E}_{\mathrm{bin}}}\right)^{1 / 5} \\
\times\left(\frac{g(e)}{\left(1-e^{2}\right)^{7 / 2}}\right)^{1 / 5} ;
\end{array}
$$

at $a_{\mathrm{GW}}$ the rate of orbital evolution due to binary-single encounters is comparable to that due to GW emission. Note that that for clusters in which dynamical ejections can be ignored, Eq. (16) and Eq. (11) show that the binary hardening rate and lifetime are independent of $m_{3}$.

In the next section we will use the model described above to address under which conditions the merger of stellar-mass BHs in a dense star cluster can lead to the rapid growth of a massive seed.

\subsection{Mass growth of a central seed}

There are (at least) two mechanisms that could lead to the ejection of BHs from a cluster: (i) binary-single interactions in which either one $\mathrm{BH}$, or both the binary and the single $\mathrm{BH}$ escape as the result of momentum conservation; and (ii) the relativistic momentum kick of the merger due to the anisotropic emission of GW radiation. We consider here an analytical model where these two mechanisms are ignored. This more tractable and idealized model will provide some important insights on the behavior of real clusters, that are independent of the assumptions made. Moreover, it is a particularly good approximation in at least two cases of interest: for clusters with large escape velocities, and for $q \ll 1$ leading to low recoil kicks.

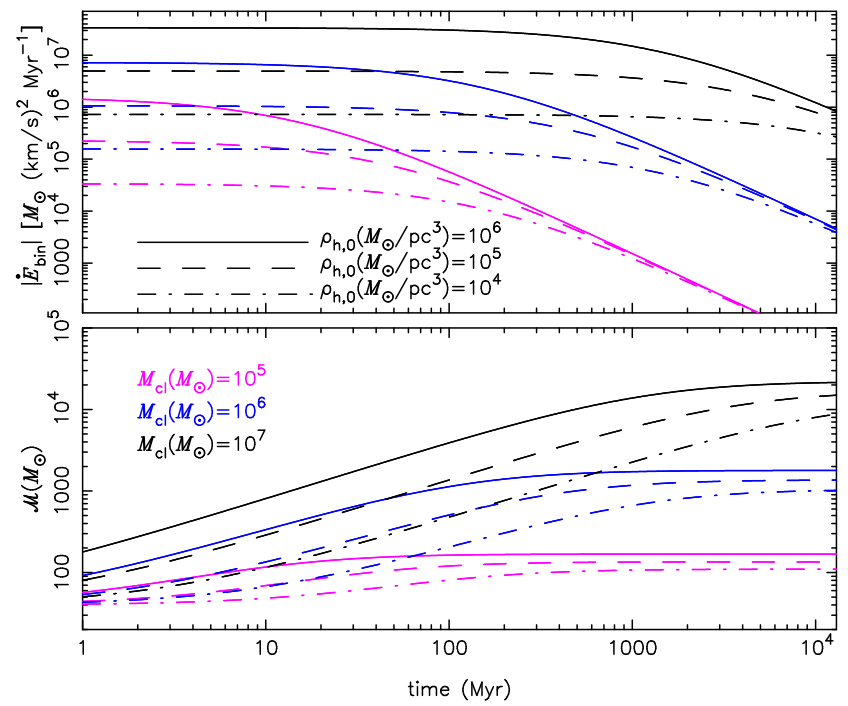

Figure 1. Time evolution of $\dot{E}$ and $\mathcal{M}$ as a function of cluster mass and density. In the bottom panel we set $m_{2}=\mathcal{M}_{0}=20 M_{\odot}$ and $e=2 / 3$ (the mean value of a thermal distribution; Jeans 1919).

If we assume that the ejection mechanism (i) can be ignored, then the overall merger rate of $\mathrm{BH}$ binaries is simply given by

$\Gamma_{\mathrm{m}} \simeq \tau_{\mathrm{bin}}^{-1}=\frac{2 a_{\mathrm{GW}}}{G m_{1} m_{2}}\left|\dot{E}_{\mathrm{bin}}\right|$.

Hence, the merger rate of $\mathrm{BH}$ binaries produced dynamically inside a massive cluster is approximately proportional to the hardening rate of the core binaries and scales with the cluster properties as $\Gamma_{\mathrm{m}} \propto M_{\mathrm{cl}}^{8 / 15} \rho_{\mathrm{h}}^{2 / 3}$. Note that the latter expression gives the $\mathrm{BH}$ merger rate even in the presence of relativistic kicks, but only until the escape velocity of the cluster is sufficiently large that mechanism (i) can be ignored, which requires $v_{\mathrm{esc}} \gtrsim 100 \mathrm{~km} \mathrm{~s}^{-1}$ (Antonini \& Rasio 2016). This condition is often met in present-day nuclear star clusters, and might also have been present in smaller clusters if they were initially much denser than observed today (see also Section 4).

A natural question to ask is whether significant growth can ever occur on a sufficiently short timescale. If we now neglect both ejection mechanisms (i) and (ii) above, then Eq. (17) leads to the following upper limit to the mass growth rate associated with a $\mathrm{BH}$ undergoing repeated mergers with smaller BHs of mass $m_{2}$ :

$\dot{\mathcal{M}}=\Gamma_{\mathrm{m}} m_{2}$.

Clearly, this latter expression should be interpreted as an upper limit to the real growth rate because we are neglecting dynamical and GW kicks. Such kicks will slow down the growth process by removing temporarily the hole from the cluster core, or halt it by fully removing the $\mathrm{BH}$ from the cluster when the recoil is sufficiently large. These additional complications will be considered in the analysis of the following section.

Integrating Eq. (18), we find the BH seed growth equa- 
tion

$$
\begin{aligned}
\mathcal{M}(t) \simeq & \mathcal{M}_{0}+\left[\frac{42}{5 G} K_{\mathrm{GW}}^{1 / 5} m_{2}^{2 / 5}\left|\dot{E}_{\mathrm{bin}, 0}\right|^{4 / 5}\right. \\
& \left.\times \frac{2 \tau_{\mathrm{rh}, 0}}{3 \zeta}\left(1-\frac{1}{\left(3 \zeta t / 2 \tau_{\mathrm{rh}, 0}+1\right)^{3 / 2}}\right)\right]^{5 / 7} .
\end{aligned}
$$

where $K_{\mathrm{GW}}=\frac{32}{5} G^{4} g(e) c^{-5}\left(1-e^{2}\right)^{-7 / 2}$, and $\mathcal{M}_{0}$ is the initial $\mathrm{BH}$ mass. The growth timescale is

$\tau_{\mathrm{M}}=\frac{2 \tau_{\mathrm{rh}, 0}}{3 \zeta}\left[\left(1-\frac{5 G\left(\mathcal{M}-\mathcal{M}_{0}\right)^{7 / 5}}{42 K_{\mathrm{GW}}^{1 / 5} m_{2}^{2 / 5}\left|\dot{E}_{\mathrm{bin}, 0}\right|^{4 / 5}} \frac{3 \zeta}{2 \tau_{\mathrm{rh}, 0}}\right)^{-2 / 3}-1\right]$.

The mass growth predicted by Eq. (19) is shown in Figure 1 as a function of cluster mass and central density (bottom panel); the upper panel of the figure gives the evolution of the binary hardening rate. From Figure 1 we see that the growth of the seed mass is interrupted soon after the relaxation driven expansion of the cluster starts, causing $\left|\dot{E}_{\text {bin }}\right|$ to decrease. The physical reason that the merger rate drops, is because the energy demand decreases, and significant drop in $\dot{a}$ happens after one initial relaxation time, which is $\propto M_{\mathrm{cl}} / \sqrt{\rho_{\mathrm{h}}}$. In fact, Eq. (19) implies that for $t \rightarrow \infty$, $\mathcal{M}(t)$ takes the finite value

$\mathcal{M}_{\max }=\mathcal{M}_{0}+110 M_{\odot} M_{5}^{23 / 21} \rho_{5,0}^{5 / 42} m_{20}^{2 / 7}$,

with $m_{20}=m_{2} / 20 M_{\odot}$. Eq. (21) demonstrates the important result that the maximum mass that can be attained via hierarchical mergers is set by the mass of the host cluster, and it is approximately independent of the cluster density, the seed mass $\mathcal{M}_{0}$ and the mass of the accreted BHs.

The model presented here leads to the following conclusions. First, Eq. (21) shows that a minimum mass $M_{\mathrm{cl}} \gtrsim$ $10^{6} M_{\odot}$ is required for the formation of a $\mathrm{BH}$ with mass $\mathcal{M} \gtrsim 10^{3} M_{\odot}$. This implies that massive clusters are the only places in which significant growth can ever occur through repeated $\mathrm{BH}$ collisions. Second, the lack of dependence on $\mathcal{M}_{0}$ and $m_{2}$ shows that the growth process should be largely independent of the the initial BH mass distribution and therefore of metallicity. These conclusions are supported by the more detailed models described in the following section, which include additional physics that we have so far ignored, e.g., dynamical friction, natal kicks, GW and dynamical recoil kicks. Including these effects increases the importance of the initial cluster density. The main results of our work are derived from these more detailed models.

\section{METHOD}

We simulate the evolution of the cluster and of its $\mathrm{BH}$ population using a semi-analytical technique. Briefly, we assume that at any time a BH binary exists in the cluster core and that this binary hardens at a rate given by Eq. (10). Thus, each binary remains in the cluster core for a time $\tau_{\text {bin }}$, and after this time a new binary is formed. We evolve the cluster $\mathrm{BH}$ population until either all $\mathrm{BH}$ s have been ejected, or until a time of $13 \mathrm{Gyr}$ has passed. Additional details of our method are given in the following.

As a first step we initialise the cluster model. This is determined by two parameters: the initial density, $\rho_{\mathrm{h}, 0}$, and the cluster mass, $M_{\mathrm{cl}}$. All time dependent quantities are derived from the analytical model of Section 2.

Then, we set the initial mass and spin distributions of the BHs. The initial spins of the BHs are drawn uniformly from the range $\chi=c J /\left(G m^{2}\right)=[0,1)$, with $J$ the spin angular momentum and $m$ the BH mass. In order to address how our results depend on the assumed spin distribution, we also explore other choices for the $\mathrm{BH}$ spins, setting their initial values all to 0 or 0.8 . When a $\mathrm{BH}$ remnant is retained and merges with new $\mathrm{BH}$ companions, we then track the evolution of its spin magnitude forward in time using the prescriptions in Rezzolla et al. (2008). We sample the masses of the stellar progenitors from a Kroupa initial mass function (Kroupa 2001) with masses in the range 20 to $100 M_{\odot}$, and evolve the stars to BHs using the Single Stellar Evolution (SSE) package (Hurley et al. 2002). We consider the lower limit of $20 M_{\odot}$ because only stars with masses larger than this produce BHs. Unless otherwise specified, we take a metallicity $Z=0.1 Z_{\odot}$. But note that for the stellar mass range considered, metallicities lower than this would all produce similar initial BH mass functions (e.g., Spera \& Mapelli 2017). Our models adopt the updated prescriptions for stellar winds and mass loss, in order to replicate the $\mathrm{BH}$ mass distribution of Dominik et al. (2013) and Belczynski et al. (2010), but include updated prescriptions for the pulsational pairinstability in massive stars (Belczynski et al. 2016). In our simulations, no $\mathrm{BH}$ can be born with a mass above $40 M_{\odot}$. Our initial sample contains a number of $\mathrm{BH}$ s equal to $0.2 \%$ of the total number of stars in the cluster (typical for a standard initial mass function; e.g., Löckmann et al. 2010). For this choice we find that about $4 \%(2 \%)$ of the total cluster mass is in $\mathrm{BHs}$ for $Z=0.1 Z_{\odot}\left(Z=Z_{\odot}\right)$.

For each $\mathrm{BH}$ we compute a natal kick velocity from a Maxwellian with dispersion $265 \mathrm{~km} \mathrm{~s}^{-1}$, as commonly done for neutron stars (Hobbs et al. 2005), and assume that the momentum imparted on a black hole is the same as the momentum given to a neutron star (Fryer \& Kalogera 2001). Thus, the natal velocity of a $\mathrm{BH}$ is lowered by the factor of $1.4 M_{\odot} / m$, with $1.4 M_{\odot}$ the typical neutron star mass. From this initial sample we remove those BHs which received a natal kick with velocity larger than $v_{\mathrm{esc}, 0}$. Using this prescription, and for the high velocity dispersion clusters we consider below $\left(v_{\text {esc }, 0} \gtrsim 70 \mathrm{~km} \mathrm{~s}^{-1}\right)$, the fraction of ejected BHs to their initial number is in all cases $\lesssim 0.01$. Note that the fraction of ejected $\mathrm{BHs}$ depends on the initial escape velocity which is related to the cluster mass and density through Eq. (6) .

At the start, we assign to each $\mathrm{BH}$ a dynamical friction time scale (Binney \& Tremaine 2011):

$\tau_{\mathrm{df}} \simeq 1.65 r_{\mathrm{h}}^{2} \frac{\sigma}{\ln \Lambda G m}=4.5 \mathrm{Myr}\left(\frac{m}{20 M \odot}\right)^{-1} M_{5} \rho_{5,0}^{-1 / 2}$

where $\sigma$ is the cluster one-dimensional velocity dispersion, and $v_{\mathrm{esc}} \simeq 4.77 \sigma$ for the adopted cluster King model. After this time has passed, a $\mathrm{BH}$ enters the core region and is allowed to form a binary with another BH. After a time $t_{\text {bin }}$ a new binary is formed assuming that the pairing probability for its BH components scales as $\propto\left(m_{1}+m_{2}\right)^{4}$, appropriate for binaries formed via three body processes (O'Leary et al. 2016). Setting the formation time for the next binary equal to the hardening timescale assures that at any time a binary exists in the centre, providing the energy needed to sustain 

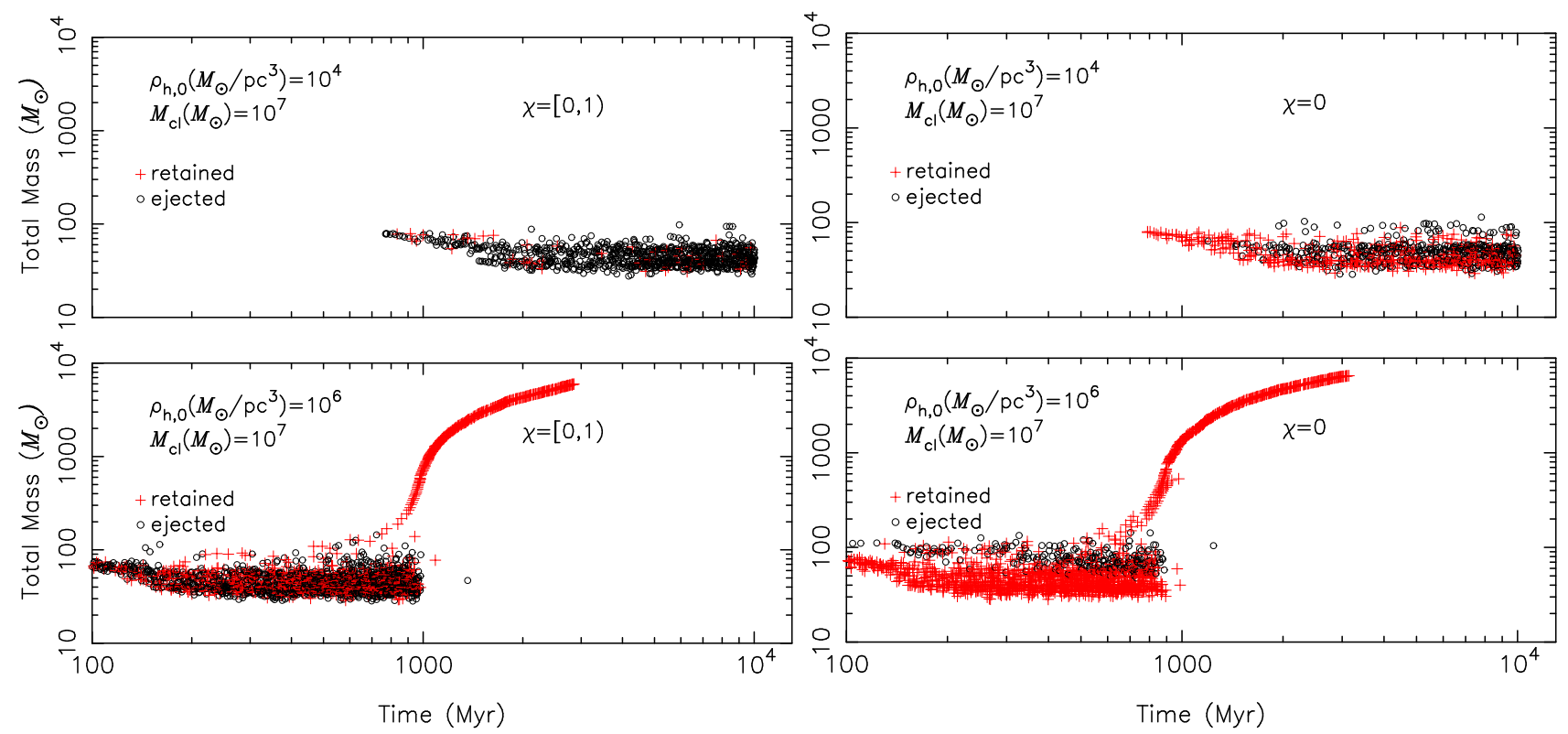

Figure 2. Mass of the merging $\mathrm{BH}$ binaries formed in four example clusters. We consider two models with the same total mass but different densities to illustrate the effect of the latter on the evolution of the BH population. In the left panels the BH spins are distributed uniformly between 0 and 1 ; in the right panels the BHs have no spin initially. Red circles are BH remnants which are retained inside the cluster, following a relativistic kick.
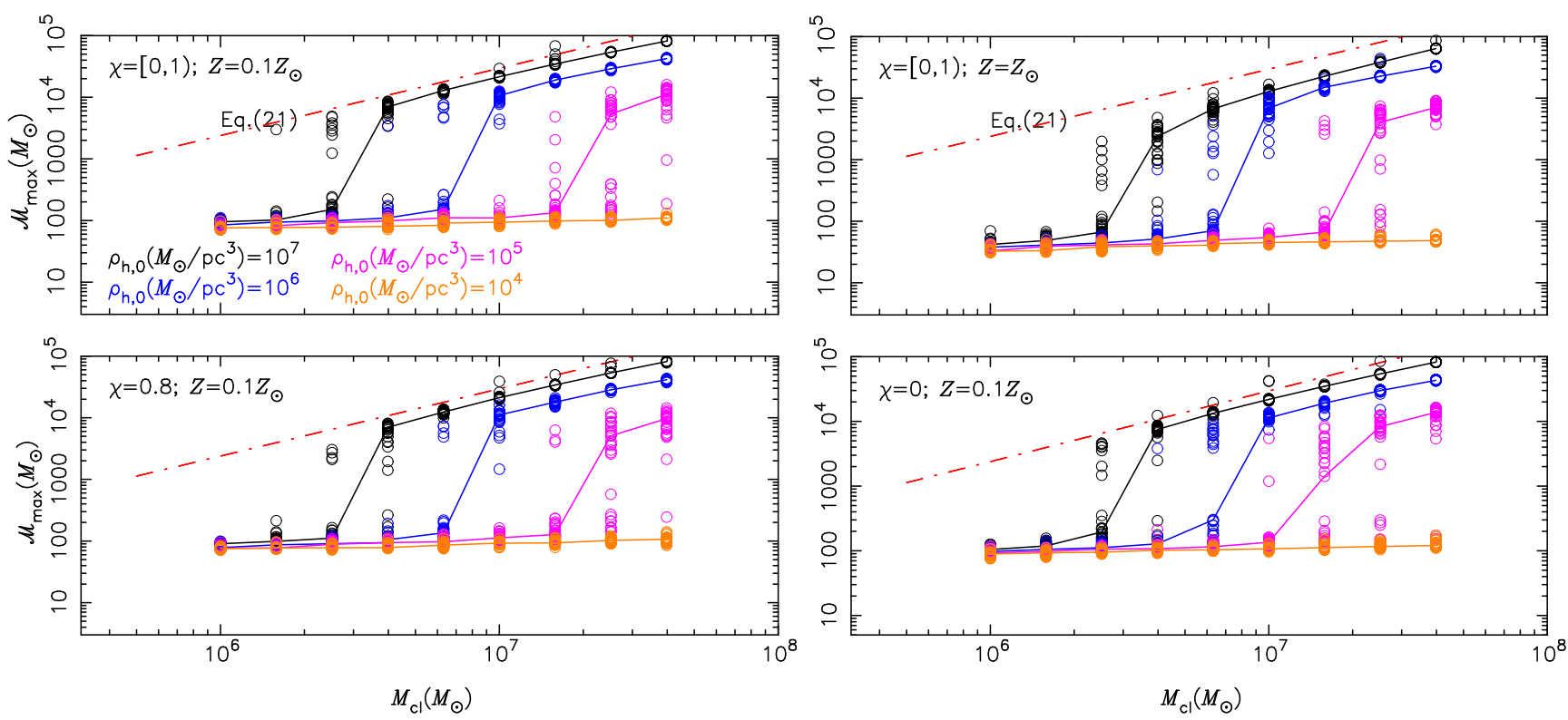

Figure 3. Maximum mass, $\mathcal{M}_{\max }$, of the merging $\mathrm{BH}$ binaries formed over $13 \mathrm{Gyr}$ of evolution, as a function of cluster properties. For each value of cluster density and mass we display the results from 30 random cluster realisations. Solid lines are median values. In the top-right we set $Z=Z_{\odot}$, and in the lower panels we set the initial BH spin magnitudes to 0.8 (left) and 0 (right). A comparison of these latter models to our fiducial set with $Z=0.1 \times Z_{\odot}$ and initial spins in the range $\chi=[0,1)$ (upper left) shows that assumptions on spins and metallicity have little effect on the value of the cluster mass and density at which substantial BH growth occurs.

the cluster core against collapse. The time from binary formation to merger is set equal to $\tau_{\text {merge }}\left(a_{\mathrm{m}}\right)=\tau_{\text {bin }}+\tau_{\mathrm{GW}}$, where $a_{\mathrm{m}}$ is obtained by sampling $e$ from a thermal distribution, $N(<e) \propto e^{2}$.

After a merger, the remnant $\mathrm{BH}$ receives a recoil kick as the result of the anisotropic emission of GW radiation with velocity $v_{\mathrm{GW}}$ (typically, $v_{\mathrm{GW}} \gg v_{2-1}$ ). The subsequent evolution of the merger remnant depends on whether it is retained or not in the cluster. We compute $v_{\mathrm{GW}}$ using the fitting formula based on the results from numerical relativity simulations of Lousto \& Zlochower (2008),

$$
\vec{v}_{\mathrm{GW}}=v_{\mathrm{m}} \hat{e}_{\perp, 1}+v_{\perp}\left(\cos \xi \hat{e}_{\perp, 1}+\sin \xi \hat{e}_{\perp, 2}\right)+v_{\|} \hat{e}_{\|},
$$

$v_{\mathrm{m}}=A \eta^{2} \sqrt{1-4 \eta}(1+B \eta)$ 

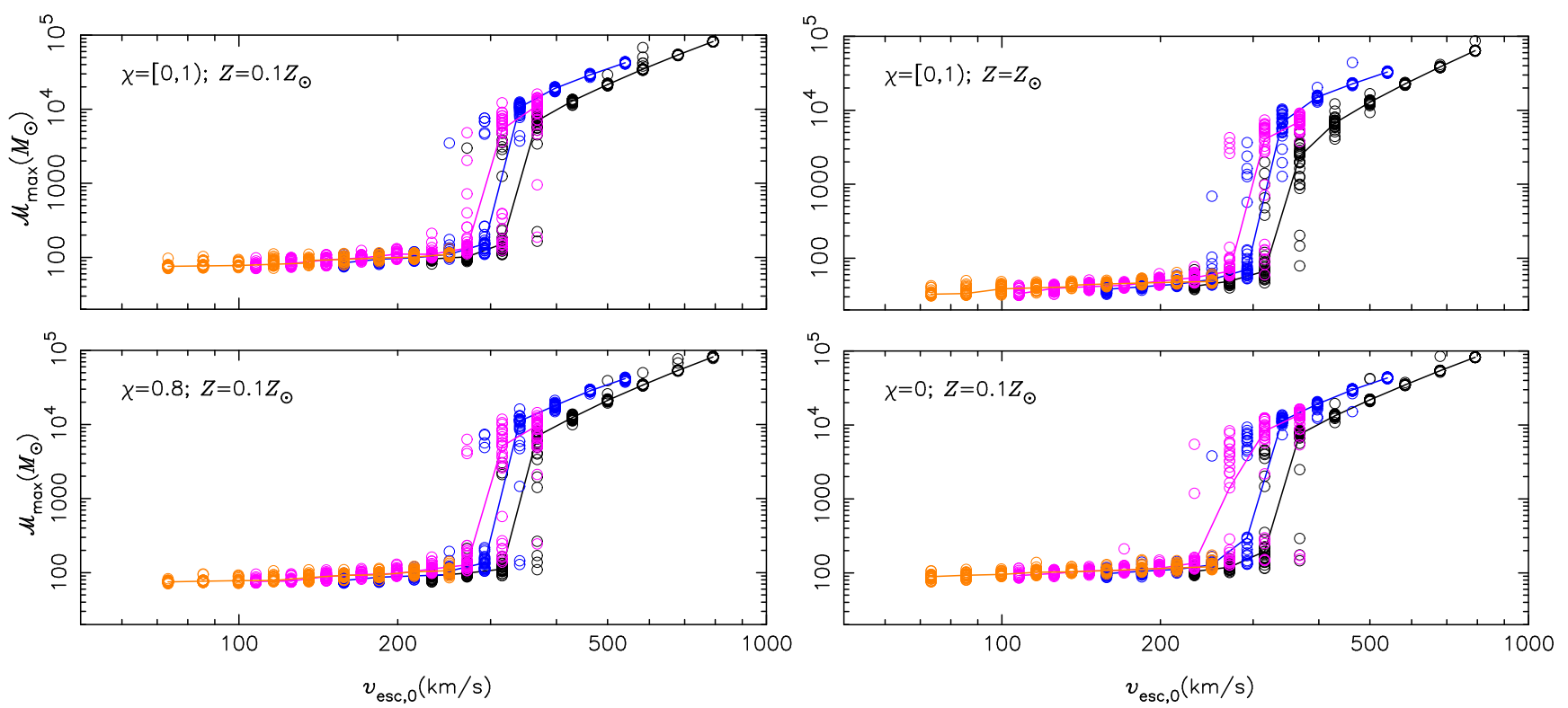

Figure 4. Similar to Fig. 3, but now the mass of the largest BH formed over 13 Gyr, $\mathcal{M}_{\max }$, is plotted as a function of cluster initial escape velocity. Different colors correspond to different densities as indicated in Fig. 3.

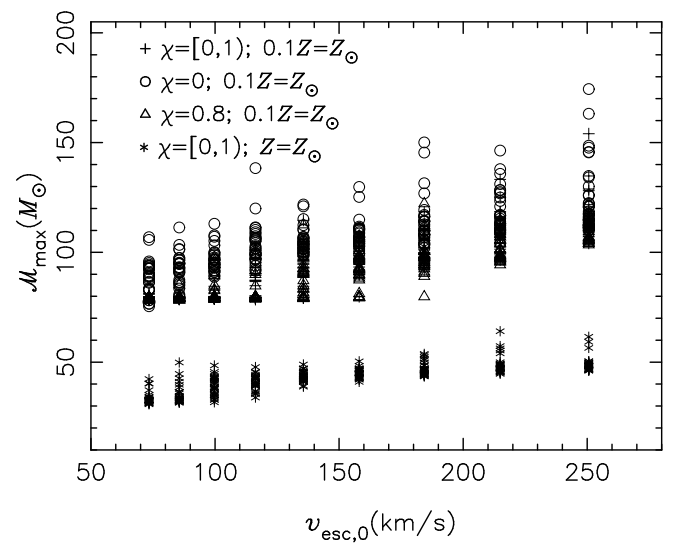

Figure 5. Zoom-in of the results displayed in Fig. 3, showing the largest $\mathrm{BHs}$ formed in clusters with initial escape velocity $\lesssim 300 \mathrm{~km} \mathrm{~s}^{-1}$ and $\rho_{\mathrm{h}, 0}=10^{4} M_{\odot} \mathrm{pc}^{-3}$. In these clusters, any growing $\mathrm{BH}$ is ejected after a few mergers, implying that the maximum mass that can be built though hierarchical mergers is affected significantly by the initial mass function of the BHs and their spins.

$v_{\perp}=\frac{H \eta^{2}}{(1+q)}\left(\chi_{2 \|}-q \chi_{1 \|}\right)$

$$
v_{\|}=\frac{16 \eta^{2}}{(1+q)}\left[V_{1,1}+V_{\mathrm{A}} \tilde{S}_{\|}+V_{\mathrm{B}} \tilde{S}_{\|}^{2}+V_{\mathrm{C}} \tilde{S}_{\|}^{3}\right] \times
$$$$
\left|\vec{\chi}_{2 \perp}-q \vec{\chi}_{1 \perp}\right| \cos \left(\phi_{\Delta}-\phi_{1}\right)
$$

where $\eta \equiv q /(1+q)^{2}$, and $q=m_{2} / m_{1} ; \perp$ and $\|$ refer to vector components perpendicular and parallel to the orbital angular momentum, respectively, and $\hat{e}_{\perp, 1}$ and $\hat{e}_{\perp, 2}$ are orthogonal unit vectors in the orbital plane. The vector $\overrightarrow{\tilde{S}} \equiv 2\left(\vec{\chi}_{2}+q^{2} \vec{\chi}_{1}\right) /(1+q)^{2}$. The values of $A=1.2 \times$ $10^{4} \mathrm{~km} \mathrm{~s}^{-1}, B=-0.93, H=6.9 \times 10^{3} \mathrm{~km} \mathrm{~s}^{-1}$, and $\xi=145^{\circ}$ are from González et al. (2007a) and Lousto \& Zlochower (2008), and $V_{1,1}=3677.76 \mathrm{~km} \mathrm{~s}^{-1}, V_{\mathrm{A}}=2481.21 \mathrm{~km} \mathrm{~s}^{-1}$, $V_{\mathrm{B}}=1792.45 \mathrm{~km} \mathrm{~s}^{-1}$, and $V_{\mathrm{C}}=1506.52 \mathrm{~km} \mathrm{~s}^{-1}$ are taken from Lousto et al. (2012). The angle $\phi_{\Delta}$ is that between the inplane component $\vec{\Delta}_{\perp}$ of the vector $\vec{\Delta} \equiv\left(m_{1}+m_{2}\right)^{2}\left(\vec{\chi}_{2}-\right.$ $\left.q \vec{\chi}_{1}\right) /(1+q)$ and the infall direction at merger. We take the phase angle $\phi_{1}$ of the binary to be random, and assume that the spin directions during mergers are isotropically distributed on the sphere. We then assign a kick velocity $v_{\mathrm{GW}}$ to the merger remnant.

The subsequent evolution has the following two possibilities: (i) $v_{\mathrm{GW}}>v_{\mathrm{esc}}$. In this case the remnant is ejected from the cluster, the remnant $\mathrm{BH}$ is removed from the sample, and a new binary is formed. (ii) $v_{\mathrm{GW}}<v_{\mathrm{esc}}$. In this case, the remnant remains in the cluster, we sum the masses of the two BHs and estimate the spin of the remnant following the prescriptions in Rezzolla et al. (2008). The remnant is deposited at a distance ${ }^{3} r_{\mathrm{in}} \simeq r_{\mathrm{h}} \sqrt{v_{\mathrm{esc}}^{4} /\left(v_{\mathrm{esc}}^{2}-v_{\mathrm{GW}}^{2}\right)^{2}-1}$, sinks back to the core on the timescale $\tau_{\mathrm{df}}\left(r_{\text {in }}\right)$ and remains "inactive" until this time has passed. After a recoil kick, the BHs will oscillate with decreasing amplitude, losing energy via dynamical friction; $\tau_{\mathrm{df}}\left(r_{\text {in }}\right)$ provides an approximation of the time over which the amplitude of the motion will fall to roughly the core radius. For a detailed treatment of this problem see Webb et al. (2018) (and references therein). After a time $\tau_{\mathrm{df}}$, the remnant is reintroduced in the core sample and is allowed to form a new binary. While the remnant stays inactive, new hard binaries are allowed to form in the core.

We terminate the integration if a time $t=13 \mathrm{Gyr}$ is reached, or all $\mathrm{BHs}$ have been removed from the cluster, in-

3 This was derived for a Plummer (1911) model, and it provides a good approximation also for moderately concentrated King models with $W_{0} \lesssim 6$. If the clusters are more centrally concentrated, then the excursions are smaller (by 1 order of magnitude for $\left.W_{0}=8\right)$ and $\tau_{\mathrm{df}}$ shorter. 

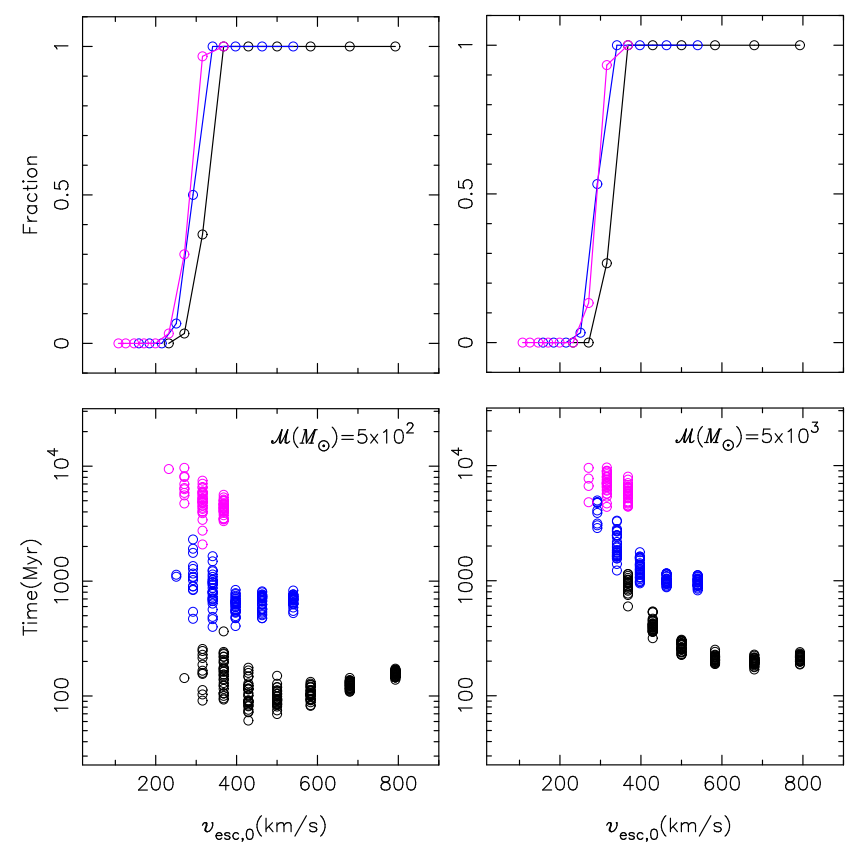

Figure 6. Upper panels show the fraction of cluster models that are able to grow a $\mathrm{BH}$ of mass $5 \times 10^{2}$ (left) and $5 \times 10^{3}$ (right) over a total of $13 \mathrm{Gyr}$ of evolution. In the lower panels we show the timescale at which these BHs are formed. For each value of cluster density and mass we display the results from 30 random cluster realisations. The figures refer to our models with $Z=0.1 Z_{\odot}$ and initial spins distributed between 0 and 1 .

cluding both the ejected binaries and the ejected interlopers. Noting that the condition for the recoil velocity experienced by a $\mathrm{BH}$ interloper to be larger than $v_{\mathrm{esc}}$ is $a \leq a_{3}=a_{\mathrm{ej}} / q_{3}^{2}$, then the total number of BHs ejected by a hard binary can be easily shown to be

$N_{3}=\int_{a_{3}}^{a_{\mathrm{m}}} \frac{1}{\epsilon-1} \frac{d a}{a}=\frac{1}{1-\epsilon} \ln \left(\frac{a_{3}}{a_{\mathrm{m}}}\right)$,

where $\epsilon=1 /(1+\delta)$. Eq. (27) shows that not only more massive clusters eject less binaries, but also the number of ejected interlopers decreases significantly.

Our numerical approach is similar to the method described in Antonini \& Rasio (2016), but with at least one important difference. Antonini \& Rasio (2016) calculated the binary hardening rate from the cluster core density which was set to be a constant free parameter in the models, and other calculations in the literature followed a similar approach (e.g., Miller \& Hamilton 2002b; Miller \& Lauburg 2009; Choksi et al. 2018). Here, we have used Hénon's principle to relate the hardening rate of the binaries to the evolving global properties of their host cluster.

\subsection{Results}

In Fig. 2 we show the mass of the merging binaries produced in four example cases of cluster evolution. We consider two cluster models with the same initial mass, but different densities. For each model we run two realisations: one where the spin parameter of the BHs is sampled from a uniform distribution in the range $[0,1)$, and the other where the BHs have no spin initially. In the model with the higher density (lower panel), due to the shorter dynamical friction timescale, the $\mathrm{BHs}$ segregate more rapidly to the cluster core and start to merge earlier during the cluster evolution. Thus, it is the value of $\tau_{\mathrm{df}}$ for the most massive $\mathrm{BHs}$ in the cluster that determines the time of the first merger (i.e., $100 \mathrm{Myr}$ in the bottom panel, and $1 \mathrm{Gyr}$ in the upper panel). Higher densities also correspond to a higher binary hardening rate and retention fraction. For $\rho_{\mathrm{h}, 0}=10^{6} M_{\odot} \mathrm{pc}^{-3}$ and initial spins in the range 0 to $1(\chi=0)$, we find that 1873 (1719) mergers are produced in the first $1 \mathrm{Gyr}$, and of the merger remnants produced over this time, 617 (1400) are retained in the cluster. For $\rho_{\mathrm{h}, 0}=10^{4} M_{\odot} \mathrm{pc}^{-3}, 959$ (915) mergers are produced over $\sim 10$ Gyrs, and only 51 (459) of the remnants are retained. Thus, higher densities lead to a higher retention fraction and faster evolution, and therefore to a larger probability that substantial growth occurs by the end of the run. Accordingly, while in the low density examples the merging binaries have masses $\mathcal{M} \lesssim 100 M_{\odot}$, for $\rho_{\mathrm{h}, 0}=10^{6} M_{\odot} \mathrm{pc}^{-3} \mathrm{a}$ BH with $\mathcal{M} \sim 10^{4} M \odot$ is formed in the first few Gyrs. As the $\mathrm{BH}$ grows, the mass ratio $q$ gets further away from unity and its dimensionless spin magnitude tends to decrease, leading to progressively lower GW kicks. This keeps the growing BH safe in the cluster after the first few mergers.

Fig. 3 shows the mass of the heaviest $\mathrm{BH}$ formed over $13 \mathrm{Gyr}$ of evolution, $\boldsymbol{M}_{\max }$, as a function of cluster properties. For each value of initial mass and density, we evolved thirty random realisations and show in the figure the mass of the largest BH formed in each run. From Fig. 3 we see that in sufficiently dense and massive clusters $\left(M_{\mathrm{cl}} \gtrsim 10^{7} M_{\odot}\right.$; $\left.\rho_{\mathrm{h}, 0} \gtrsim 10^{5} M_{\odot} \mathrm{pc}^{-3}\right)$, BHs mergers can lead to substantial growth and produce massive seeds. Fig. 3 shows that for the range of stellar densities we considered $\rho_{\mathrm{h}, 0} \leq 10^{7} M_{\odot} \mathrm{pc}^{-3}$, only clusters with total mass larger than several $10^{6} M_{\odot}$ produce massive seeds.

In Fig. 4 we plot $\mathcal{M}_{\max }$ as a function of initial cluster escape velocity. There is a clear transition near a cluster escape velocity of $v_{\text {esc, } 0}=300 \mathrm{~km} \mathrm{~s}^{-1}$. Clusters with an initial escape velocity larger than this value are able to grow BHs with masses above $1000 M_{\odot}$ in less than a Hubble time. Assumptions about metallicity and initial spins have little or no effect on this conclusion. The transition near $v_{\text {esc }, 0}=300 \mathrm{~km} \mathrm{~s}^{-1}$ is explained mainly from the fact that the mean value of the GW kick velocity distribution is around $300 \mathrm{~km} \mathrm{~s}^{-1}$ for large spins (see Fig. 1 in Amaro-Seoane \& Chen 2016).

For lower escape velocities, i.e., $v_{\mathrm{esc}}<300 \mathrm{~km} \mathrm{~s}^{-1}$, one finds that assumptions about spins and metallicity affect the results in important ways (see Fig. 5). In these clusters, any growing $\mathrm{BH}$ is eventually ejected after a few mergers, so that the maximum mass that can be built though hierarchical mergers depends significantly on the initial mass function of the $\mathrm{BHs}$ and their initial spins. If the $\mathrm{BHs}$ have initially high spins, the mass of the largest $\mathrm{BH}$ formed is about twice the high mass end of the $\mathrm{BH}$ progenitor population. Thus, its value depends on metallicity. For $Z=0.1 Z_{\odot}$, $\mathcal{M}_{\max } \approx 100 M_{\odot}$; for $Z=Z_{\odot}, \mathcal{M}_{\max } \approx 50 M_{\odot}$. If the BHs are formed with no spin, some fraction of the merger remnants will be retained due to the lowered GW recoil. This second generation $\mathrm{BHs}$, however, are formed with a large spin $\chi \simeq 0.7$, and they will be most likely ejected if they undergo a second merger. It follows that the mass of the largest BH 


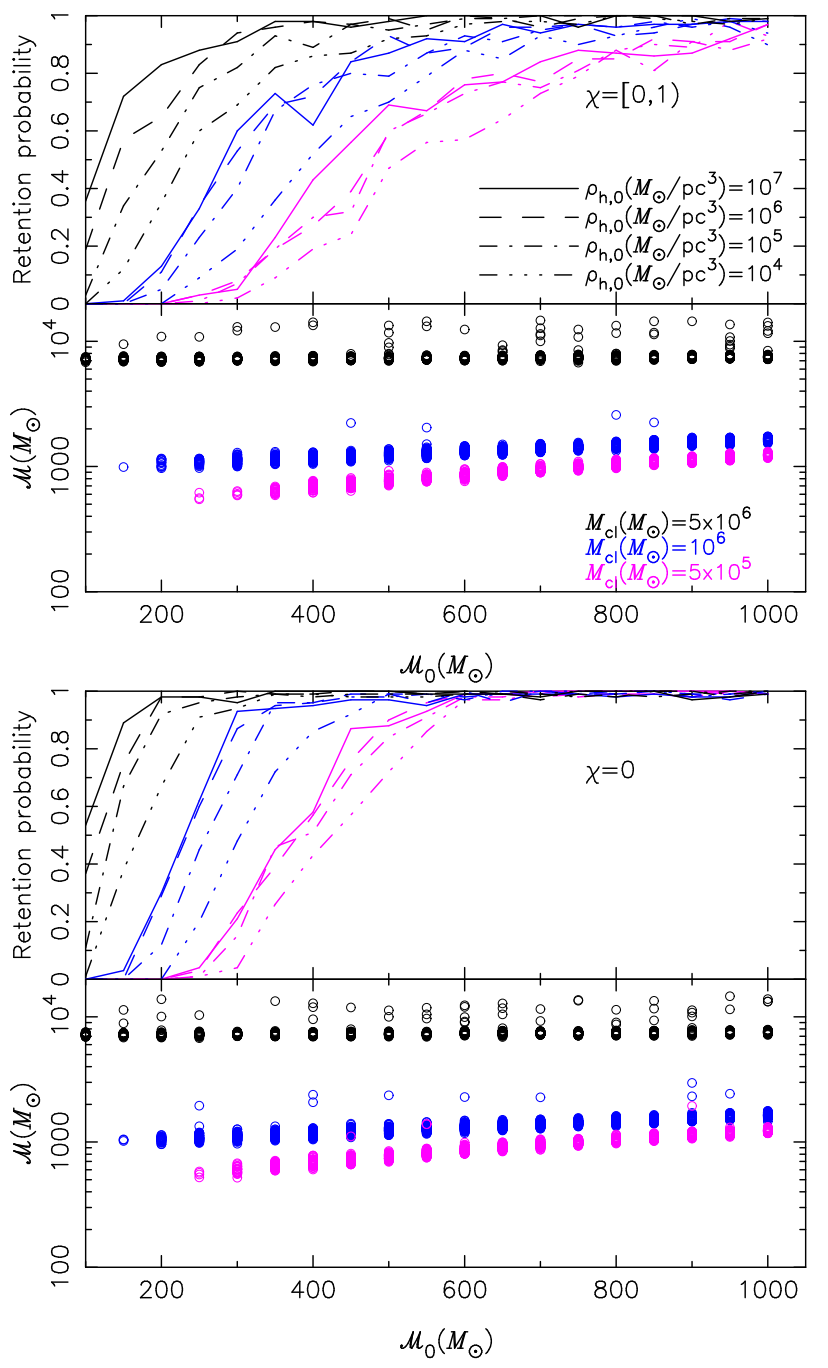

Figure 7. The upper panel in each figure gives the probability that a $\mathrm{BH}$ of initial mass $\mathcal{M}_{0}$ will still be inside its parent cluster after 13 Gyr of evolution. Cluster initial parameters are indicated; we set $Z=0.1 Z_{\odot}$, and vary $\mathcal{M}_{0}$ in the range $[100 ; 1000] M_{\odot}$. For each cluster mass and density we simulated 100 models. The lower panels show the mass of the retained $\mathrm{BH}$ seeds at the end of the integration. Upper figure is for initial BH spin magnitudes distributed in the range $\chi=[0,1)$; in the lower figure instead, the $\mathrm{BHs}$ have zero spin initially.

that can be formed through repeated mergers in this case is about four times the mass of the largest $\mathrm{BH}$ that is produced by stellar evolution. Accordingly, for $Z=0.1 Z_{\odot}$ and non spinning $\mathrm{BHs}$, our models do not typically produce $\mathrm{BHs}$ more massive than about $150 M_{\odot}$ (see Fig. 5).

Our results appear to be in good agreement with those of Rodriguez et al. (2018). These authors investigated the formation of second generation $\mathrm{BHs}$ in their Monte Carlo models of globular clusters. Thus the comparison we make here only concerns systems similar to globular clusters $\left(v_{\text {esc, }, 0} \lesssim 100 \mathrm{~km} \mathrm{~s}^{-1}\right)$. Rodriguez et al. (2018) found BH masses as high as $\simeq 150 M_{\odot}$, but only get to $80 M_{\odot}$ once the spins were increased (see their Fig. 2).

Amaro-Seoane \& Chen (2016) predicted that BH binaries observed by ground-based detectors should have similar masses, low spin magnitudes and zero eccentricities, regard- less of how they have formed. Thus, since current detectors only observe a certain subset of all mergers they cannot distinguish formation mechanisms. The same authors later showed that a decihertz observatory could instead distinguish formation mechanisms as the eccentricity imprint from the formation channels is measurable at lower frequencies (Chen \& Amaro-Seoane 2017). We agree with these authors that the bulk of the $\mathrm{BH}$ merger populations produced by field and dynamical channels should have similar properties in the LIGO/Virgo frequency range, and that repeated mergers are unlikely to occur in any cluster due to the large escape velocities required. However, our models also show that the detection of a $\mathrm{BH}$ binary with total mass in the range $\simeq 100-200 M_{\odot}$ will immediately imply dynamical formation through hierarchical mergers in a nuclear star cluster. Field formation (from both binaries and triple stars) and dynamical formation in globular clusters cannot produce $\mathrm{BH}$ binaries with total masses in this range.

Finally, in Fig. 6 we present predictions for the BH growth timescale (bottom panels), and the fraction of our models in which a $\mathrm{BH}$ of a given mass is formed after $13 \mathrm{Gyr}$ of evolution (upper panels). The probability that substantial growth will occur in a cluster model appears to be a strong function of the cluster escape velocity. The formation probability is one for $v_{\mathrm{esc}, 0} \gtrsim 300 \mathrm{~km} \mathrm{~s}^{-1}$, and it drops sharply to zero for escape velocities smaller than this value. The timescale over which the growth of the seed occurs is most sensitive to the cluster density. We find that our cluster models with the highest densities (i.e., $\rho_{\mathrm{h}, 0} \geq 10^{6} M_{\odot} \mathrm{pc}^{-3}$ ), are able to grow a $\mathrm{BH}$ with mass $\mathcal{M} \gtrsim 10^{3} M_{\odot}$ in a time $\lesssim 1 \mathrm{Gyr}$, while for $\rho_{\mathrm{h}, 0}=10^{5} M_{\odot} \mathrm{pc}^{-3}$ it takes a time $\sim 10 \mathrm{Gyr}$ to grow a comparable massive seed.

An approximate value of the minimum cluster density that is needed in order for mass growth to be able to occur is obtained by requiring that the following two conditions are met: (i) $v_{\mathrm{esc}, 0}>300 \mathrm{~km} \mathrm{~s}^{-1}$, and (ii) the $\mathrm{BH}$ dynamical friction timescale is shorter than a Hubble time, i.e. $t_{\mathrm{df}}\left(r_{\mathrm{h}}\right)<$ 13 Gyr. Using Eq.(6) and Eq.(22) we find that conditions (i) and (ii) cannot be contemporarily satisfied for $\rho_{\mathrm{h}, 0} \lesssim$ $10^{4} M_{\odot} \mathrm{pc}^{-3}\left(m / 20 M_{\odot}\right)^{-1}$, and therefore any BH mass growth should be suppressed in clusters with densities lower than this value. This conclusion appears to be in agreement with the results shown in Fig. 6 where we see that for $\rho_{\mathrm{h}, 0}=$ $10^{5} M_{\odot} \mathrm{pc}^{-3}$ it takes a time $\sim 10 \mathrm{Gyr}$ to grow any massive seed. For densities smaller than this, any BH mass growth is suppressed.

The main take away from the analysis presented in this section is that in clusters with initial escape velocity $\gtrsim 300 \mathrm{~km} \mathrm{~s}^{-1}$ and averaged density $\gtrsim 10^{5} M_{\odot} \mathrm{pc}^{-3}$, repeated $\mathrm{BH}$ mergers should lead to the formation of BHs with mass above $10^{3} M_{\odot}$ in less than a Hubble time.

\subsection{Clusters with a primordial massive seed}

In our models, the initial mass function of the BHs has a natural upper mass limit of about $40 M_{\odot}$. This limit is set by the pulsational pair instability supernovae (e.g., Woosley et al. 2007; Chen et al. 2014), and pair instability supernovae (Barkat et al. 1967). If the mass of the Helium core is $\gtrsim 30 M_{\odot}$, the formation of electron-positron pairs makes oxygen/silicon burn explosively. Hydrodynamical simulations 
show that if the helium core mass is $M_{\mathrm{He}} \lesssim 64 M_{\odot}$ the star experiences several pulses that enhance mass loss before the star forms a compact remnant, while in the range $64 \lesssim M_{\mathrm{He}} \lesssim 135 M_{\odot}$ the oxygen/silicon ignition releases enough energy to disrupt the entire star (Woosley 2017). For $M_{\mathrm{He}} \gtrsim 135 M_{\odot}$, however, the star is expected to avoid the pair instability and directly collapse to a massive remnant. Spera \& Mapelli (2017) show that very massive stars with initial mass $\gtrsim 200 M_{\odot}$ and $Z<0.05 Z_{\odot}$ form $\mathrm{BHs}$ with mass above $\gtrsim 200 M_{\odot}$ via direct collapse. This means that, although rarely, some clusters might form an initial massive seed well before BH mergers can play any important role. Gieles et al. (2018b) show that stars above $300 M_{\odot}$ can also form via stellar collisions in the first few Myrs of evolution of a dense globular cluster.

After its formation, a massive seed will inevitably merge with other BHs in the core, and following each merger it will receive a GW recoil kick. Although such kicks will be somewhat reduced by the smaller mass ratios involved (see Eq. 23), they can still be sufficiently large to eject the seed from the cluster (Mandel et al. 2008; Holley-Bockelmann et al. 2008; Konstantinidis et al. 2013; Fragione et al. 2018a,b). Here we calculate the probability for this to happen. We consider cluster models with various masses and densities, in which we introduced an initial $\mathrm{BH}$ with mass in the range $100 \leq \mathcal{M}_{0} \leq 1000 M_{\odot}$. For each seed mass we integrated 100 cluster models with a given density and mass, and set $Z=0.1 Z_{\odot}$. We evolve these models for $13 \mathrm{Gyrs}$, and plot in Fig. 7 the fraction of systems in which the seed is still in the cluster after this time (upper panels). In the lower panels of Fig. 7, we plot the final mass of the seeds.

Fig. 7 shows that whether a seed will be kept inside its parent cluster or not depends on the cluster mass. For clusters with mass $\sim 10^{6} M_{\odot}$, an initial seed mass of $M_{0} \gtrsim 200 M_{\odot}$ is needed for the retention probability to be significant. The lower panels in Fig. 7 show that the mass of the growing seeds at the end of the evolution is essentially independent of the initial seed mass, and it is almost exclusively determined by the cluster mass, in agreement with Eq. (21) and our discussion in Section 2.1. On average, the value of the final $\mathrm{BH}$ seed mass was also found to be independent of the initial cluster density.

\subsection{Approximations and assumptions}

Our models are based on a number of assumptions which we now discuss and justify in more detail.

We have assumed that the dynamical interactions only occur between BHs. This is reasonable because for an evolved cluster, the densities of BHs near the core are expected to be much larger than the densities of stars. Hence, $\mathrm{BHs}$ will dominate the interactions (e.g., Breen \& Heggie 2013; Morscher et al. 2015). Moreover, because close threebody interactions pair the $\mathrm{BHs}$ with the highest mass and tens of three body encounters are required before merger, then mergers will be primarily between $\mathrm{BHs}$ when they are present (e.g., Sigurdsson \& Hernquist 1993; Gültekin et al. 2004; Miller \& Lauburg 2009). If the number of BHs in the core is low, then they could exchange more frequently in mass-transferring binaries with main sequence, giant, and white dwarf companions (e.g., Kremer et al. 2018), which can lead to an observable electromagnetic signature (e.g.,
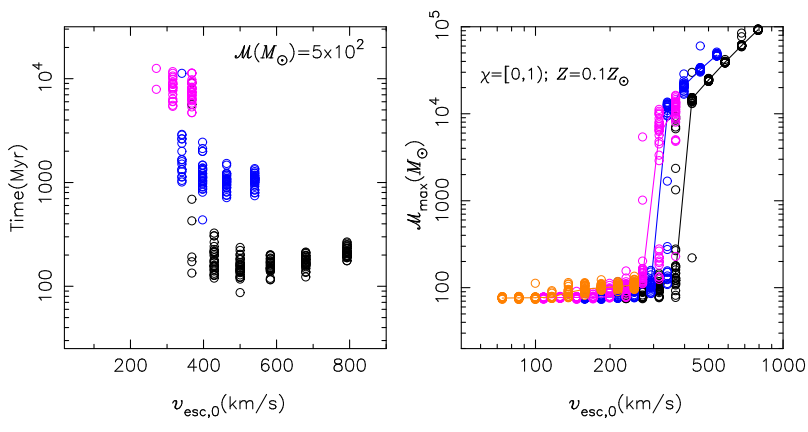

Figure 8. The left panel shows the timescale needed to grow a seed with mass above $5 \times 10^{2} M_{\odot}$, and the right panel gives the maximum mass of the binary $\mathrm{BHs}$ formed as a function of the cluster initial escape velocity. Unlike our other models, in these simulations we have included the time delay due to the wandering of the $\mathrm{BH}$ seed off the cluster centre caused by threebody superelastic scatterings. A comparison to Fig. 4 (top-left panel) and Fig. 6 (bottom-left panel) shows that this effect does not represent a limiting factor for the growth of a massive seed.

Strader et al. 2012). It should be also noted that in the most relevant case in which dynamical ejections can be ignored (i.e., $v_{\mathrm{esc}} \gg v_{2-1}$ ), then the binary hardening rate and merger time are only determined by the cluster properties and are independent of the interloper mass (see also Section 2).

Another simplification we made is to neglect the relativistic corrections of the orbits during binary singleinteractions. The most important corrections are the $2.5 \mathrm{pN}$ terms, which for $\lesssim 5 \%$ of the systems could lead to a faster merger than predicted by our models. For a detailed discussion of these effects see Gültekin et al. (2006) and Samsing (2018).

Finally, we have set the binary merger time equal to $\tau_{\text {merge }}=\tau_{\text {bin }}+\tau_{\mathrm{GW}}$. But the merger time will be exactly given by $\tau_{\text {merge }}$ only if the binary remains confined near the core region so that the rate of binary-single interactions does not drop significantly below the value given by Eq. (10). This might not be a good approximation at early stages when the seed is "light" and can be kicked off the centre due to three body interactions, causing the hardening rate to go down or to nearly stop (MacLeod et al. 2016; de Vita et al. 2018). However, the energy balance argument implies that the overall merger rate of binaries should be hardly affected by this - if the binary spends a significant amount of time outside the core region a new binary must form in the core in order to generate the required heating rate, leaving the $\mathrm{BH}$ merger rate unaffected. Furthermore, the displacement due to the GW recoils (which are included in our models above) and the resulting time delay are typically much larger than those due to superelastic three-body encounters which therefore should have a secondary effect on the growth timescale of the seed.

To demonstrate the latter point, we ran a new suit of simulations in which the time the binary spends outside the core was included in the calculation of its merger time. Following the numerical procedure described in Samsing \& D'Orazio (2018), we divide the dynamical evolution of a binary in $N_{2-1}$ isolated binary-single interactions that lead to a stepwise decrease in the binary semi-major 
axis until $a \approx a_{\mathrm{m}}$. Following the $i^{\text {th }}$ interaction, the binary semi-major axis decreases from $a^{i}$ to $\epsilon a^{i}$. The binary centre of mass receives a kick $v_{2-1}$ and it is deposited at a distance $r_{\text {in }}\left(v_{2-1}\right)$ from the centre. Given the new binary orbit, we compute the time for the next interaction to occur as $\tau_{\mathrm{df}}^{i}\left(r_{\mathrm{in}}\right)+\tau_{\mathrm{enc}}^{i}$, where $\tau_{\text {enc }}^{i} \simeq 0.2 \frac{G m_{1} m_{2}}{2 \epsilon a^{i}} \dot{E}_{\text {bin }}^{-1}$ and $\dot{E}_{\text {bin }}$ is computed at time $t+\tau_{\mathrm{df}}^{i}$. The total lifetime of the binary is then $\tau_{\text {merge }}=\tau_{\mathrm{GW}}+\sum_{i}^{N_{2-1}}\left(\tau_{\mathrm{df}}^{i}+\tau_{\mathrm{enc}}^{i}\right)$. We consider models with $Z=0.1 Z_{\odot}$ and initial $\mathrm{BH}$ spins sampled uniformly in the range $\chi=[0,1)$. The results from these new models are presented in Fig. 8. A comparison with the results from the simulations described above (see Fig. 4 and Fig. 6) confirms that accounting for the delay time due to dynamical kicks has a small effect on the growth timescale and final mass of the seed.

\section{ASTROPHYSICAL IMPLICATIONS}

\subsection{Globular clusters}

A number of studies have argued that ground based GW observations will be able to address whether intermediate mass BHs (IMBHs; generally defined as $\mathrm{BHs}$ with mass in the range $\sim 100-10^{5} M_{\odot}$ ) can form in globular clusters via hierarchical mergers of stellar seed BHs (e.g., Miller \& Hamilton 2002c; Gültekin et al. 2004; Giersz et al. 2015; Kovetz et al. 2018; Christian et al. 2018). Our dynamical models show, however, that in clusters with parameters consistent with present-day globular clusters $\left(M_{\mathrm{cl}} \lesssim 10^{7} M_{\odot}\right.$, $\left.\rho_{\mathrm{h}} \lesssim 10^{5} M_{\odot} / \mathrm{pc}^{3}\right)$, it is not possible to form BHs above $\approx$ $100 M_{\odot}$ through mergers of smaller BHs (see Fig. 9), because their initial escape velocities are too low $\left(\lesssim 300 \mathrm{~km} \mathrm{~s}^{-1}\right)$. This result appears to be rather insensitive to the assumptions about dynamics and about the initial distribution of spins and masses of the BHs. On the other hand, globular clusters could have formed a massive $\mathrm{BH}$ via mergers in the past if their escape velocities were initially much larger than today; for example, a cluster mass of $M_{\mathrm{cl}} \lesssim 10^{6} M_{\odot}\left(10^{5} M_{\odot}\right)$ would require an initial half-mass radius $r_{\mathrm{h}, 0} \lesssim 0.1 \mathrm{pc}(0.01 \mathrm{pc})$. As shown in Fig. 9 such extremely compact clusters will then expand within a Hubble time to become the larger clusters we observe today.

We conclude that if future observations will show that IMBHs exist at the centre of globular clusters, then the scenario presented here could be a plausible explanation to their origin and would imply that these systems form at high redshift with extremely large densities ( $\gtrsim 10^{7} M_{\odot} \mathrm{pc}^{-3}$ ). Other scenarios, invoking runaway collisions of massive stars and/or the collapse of a very massive $\operatorname{star}\left(\gtrsim 200 M_{\odot}\right)$ could also lead to the formation of IMBHs in globular clusters (e.g., Portegies Zwart \& McMillan (2002), Gürkan et al. (2004), Portegies Zwart et al. (2004a), Portegies Zwart et al. (2004b), Freitag et al. (2006), Mapelli (2016), Gieles et al. (2018b), and Section 3.2). A GW detection may be the most promising route to search for IMBHs in globular clusters, because a convincing detection with other methods remains elusive (Anderson \& van der Marel 2010; Lanzoni et al. 2013; Gieles et al. 2018a; Tremou et al. 2018; Amaro-Seoane 2018b).

\subsection{Nuclear star clusters}

Nuclear star clusters (NCs) are the most massive and densest clusters observed in the local universe (Carollo et al. 1997; Böker et al. 2004; Côté et al. 2006). Miller \& Lauburg (2009) and Antonini \& Rasio (2016) argued that in most NCs, due to their large escape velocities, dynamical ejections can be reasonably ignored. Under these conditions, Eq. (17) gives the total $\mathrm{BH}$ merger rate produced by a cluster of a given mass and radius, and can be used to determine the total merger rate per volume produced locally by NCs:

$\Gamma_{\mathrm{tot}} \simeq \frac{d N_{g x}}{d V} f_{\mathrm{BH}} \frac{1}{N_{\mathrm{NC}}} \sum_{i=1}^{N_{\mathrm{NC}}} \Gamma_{\mathrm{m}, i}\left(M_{\mathrm{cl}}, r_{\mathrm{h}}\right)$

where $\Gamma_{\mathrm{m}}$ is the BH merger rate given by Eq. (17), $N_{\mathrm{NC}}$ is the total number of clusters in the sample considered, $d N_{g x} / d V$ is the number density of nucleated galaxies in the local universe, and $f_{\mathrm{BH}}$ is the fraction of these galaxies without a massive $\mathrm{BH}$. Because the value of $f_{\mathrm{BH}}$ is poorly constrained by observations, we leave it as a parameter in our calculations below, but note that values of $f_{\mathrm{BH}} \gtrsim 0.5$ are consistent with the results of galaxy formation models (Antonini et al. 2015), while observations prefer marginally smaller values $f_{\mathrm{BH}} \sim 0.2$ (Seth et al. 2008; Nguyen et al. 2018). Most probably $f_{\mathrm{BH}}$ should also be a function of galaxy mass and morphology, which we ignore here. We assume that the BHs have all the same mass which we take to be $10 M_{\odot}$. We set $d N_{g x} / d V=0.01 \mathrm{Mpc}^{-3}$ (Conselice et al. 2005) and assume an order of unity occupation fraction of NCs in galaxies, (Turner et al. 2012; Georgiev \& Böker 2014).

Because the merger rate is determined at any given time by the current cluster properties through Eq. (17), it is not affected by the details of how the cluster has evolved and formed at earlier times nor on the galaxy morphology and mass. Thus, in order to compute the local merger rate of $\mathrm{BH}$ binaries produced by a population of NCs we only need to know their present-day values of $r_{\mathrm{h}}$ and $M_{\mathrm{cl}}$, assuming that these clusters contain BHs. We compute $\Gamma_{\mathrm{m}}\left(M_{\mathrm{cl}}, r_{\mathrm{h}}\right)$ for the $N_{\mathrm{NC}}=151$ clusters in the observational sample of Georgiev et al. (2016) with a well determined mass and effective radius (see Fig. 9).

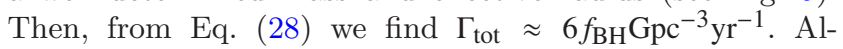
though necessarily approximated, this calculation shows that NCs (without a massive $\mathrm{BH}$ ) contribute significantly to the overall merger rate of binary $\mathrm{BHs}$ in the local universe (see also Antonini \& Rasio 2016, for a similar result). Including the contribution from NCs hosting a massive $\mathrm{BH}$ could increase these rates significantly (O'Leary et al. 2009; Antonini \& Perets 2012; VanLandingham et al. 2016; Petrovich \& Antonini 2017; Hamers et al. 2018).

We showed that clusters with escape velocity $v_{\mathrm{esc}, 0} \gtrsim$ $300 \mathrm{~km} \mathrm{~s}^{-1}$ and initial densities $\gtrsim 10^{5} M_{\odot} \mathrm{pc}^{-3}$, can grow a $\mathrm{BH}$ with mass well above $\approx 100 M_{\odot}$ in less than a Hubble time. Such higher densities and escape velocities lead to higher retention fractions and merger rates, favouring rapid growth. Now, we turn to the question of whether such extreme conditions are met in NCs. Fig. 9 gives the total mass and half-light radius (or effective radius) of the NCs in the sample of Georgiev et al. (2016). This sample comprises the NCs in spheroid-dominated galaxies from Côté et al. (2006) and Turner et al. (2012), and disk dominated galaxies from Georgiev et al. (2009) and Georgiev \& Böker (2014). The 


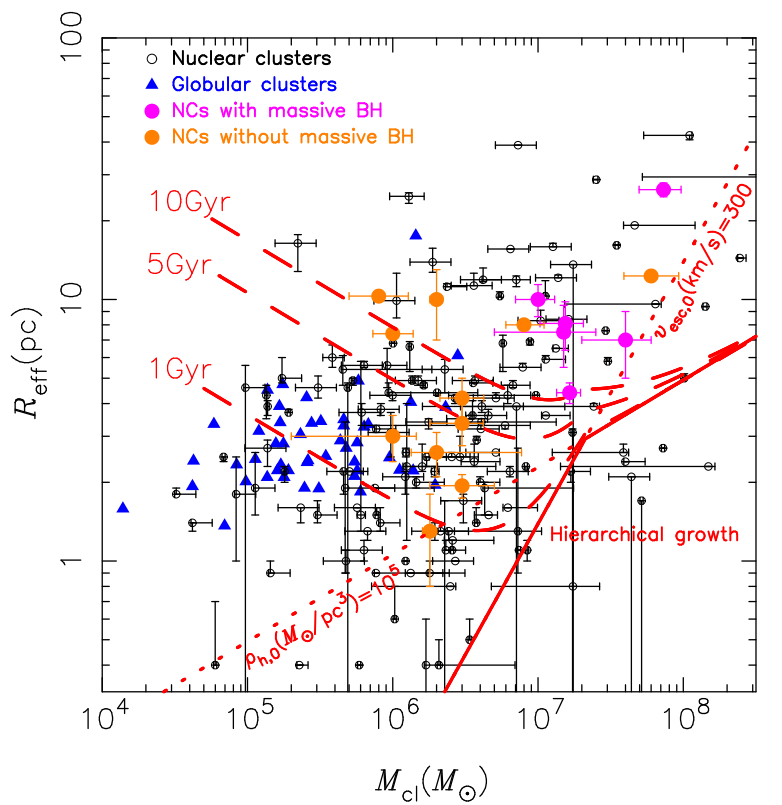

Figure 9. Effective radii and masses of NCs from Georgiev et al. (2016), and globular clusters from Forbes et al. (2008). Below the solid-red line our models predict that an IMBH will grow in less than a Hubble time. The dashed-red lines show the effective radius of clusters with initial density $\rho_{\mathrm{h}, 0}=10^{5} M_{\odot} \mathrm{pc}^{-3}$ and escape velocity $v_{\text {esc }, 0}=300 \mathrm{~km} \mathrm{~s}^{-1}$ after evolving them for 1,5 and $10 \mathrm{Gyr}$ (from bottom to top line). If we assume mass follows light, then $r_{\mathrm{h}} \simeq 4 / 3 R_{\text {eff }}$. Purple symbols are systems for which a central massive BH has been measured. Orange symbols are NCs for which there is only an upper limit to the central BH mass. See text for additional details.

range of plausible parameters for which, according to our previous analysis, hierarchical growth is likely to occur are indicated by the box in the bottom-right corner of the figure. This region of parameter space is defined as that where both conditions $v_{\mathrm{esc}, 0} \gtrsim 300 \mathrm{~km} \mathrm{~s}^{-1}$ and $\rho_{\mathrm{h}, 0} \gtrsim 10^{5} M_{\odot} \mathrm{pc}^{-3}$ are met. Remarkably, we find that 17 out of the 151 of the NCs in the sample have structural parameters that are consistent with being inside this region of parameter space. Hence a fraction $f_{\mathrm{NC}} \approx 0.1$ of the present-day NCs meet the conditions required for fast hierarchical $\mathrm{BH}$ mergers to occur within their cores. If present day NCs with the required properties exist today, then they must have been more abundant in the early Universe when densities where higher. This motivates searches for IMBHs with mass modelling approaches in present day NCs (that do not longer have the required densities).

If several $\mathrm{BHs}$ with masses $>100 M_{\odot}$ are formed, these will provide strong sources of GWs detectable by Advanced LIGO/Virgo through their merger and/or ringdown (e.g., Miller 2002; Veitch et al. 2015). We compute the corresponding merger rate per volume as

$\Gamma_{\mathrm{V}} \simeq \frac{d N_{g x}}{d V} f_{\mathrm{BH}} f_{\mathrm{NC}} \frac{1}{N_{\mathrm{NC}}} \sum_{i=1}^{N_{\mathrm{NC}}} \Gamma_{\mathrm{m}, i}\left(M_{\mathrm{cl}}, r_{\mathrm{h}}\right)$

where $f_{\mathrm{BH}}$ should be intended here as the fraction of NCs in the relevant region of parameter space that do not have a massive $\mathrm{BH}$, and $\Gamma_{\mathrm{m}, i}\left(M_{\mathrm{cl}}, r_{\mathrm{h}}\right)$ the merger rate of $\mathrm{BHs}$ in the mass range $\mathcal{M}=\left[10^{2}, 10^{3}\right] M_{\odot}$. We compute the sum in
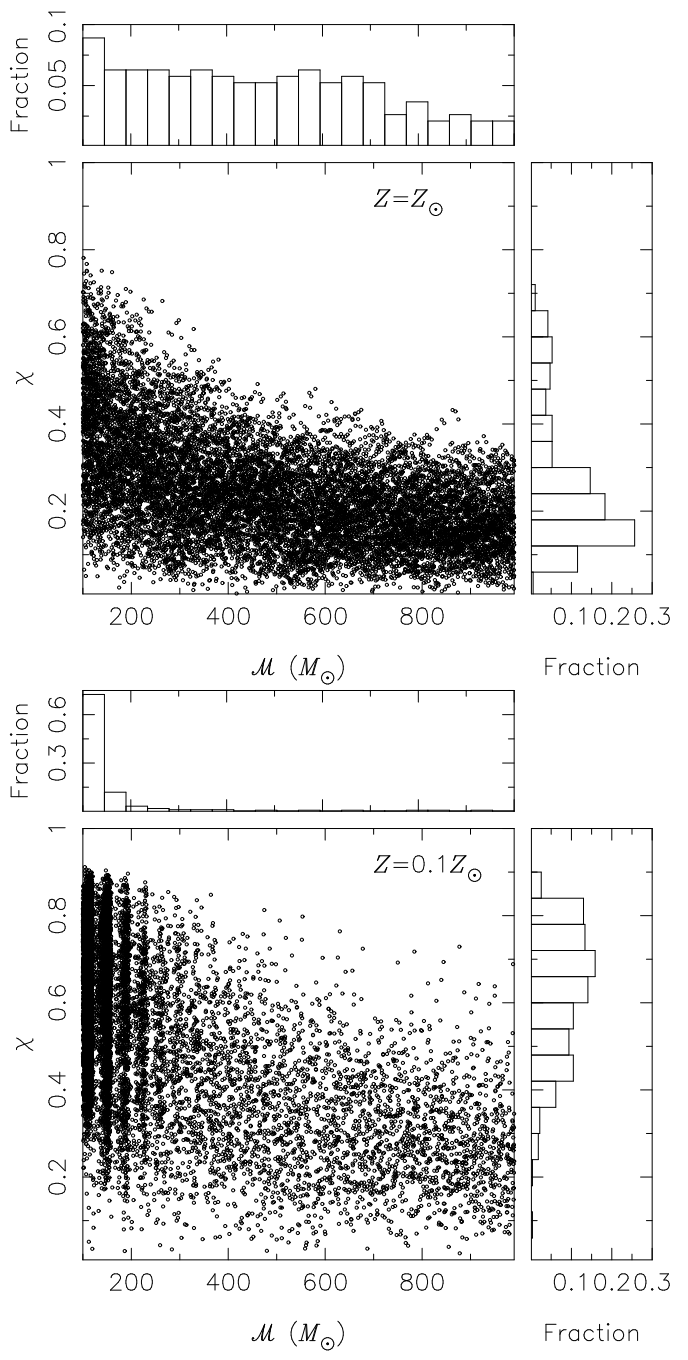

Figure 10. Intrinsic spin and mass distributions for the merging binaries with mass $\mathcal{M}>100 M_{\odot}$ formed in our cluster models (cluster radius and mass were uniformly distributed inside the region where hierarchical growth is possible, i.e., $v_{\mathrm{esc}, 0} \geq 300 \mathrm{~km} \mathrm{~s}^{-1}$ and $\left.\rho_{\mathrm{h}, 0} \geq 10^{5} M_{\odot} \mathrm{pc}^{-3}\right)$. Upper panel is for Solar metallicities, lower panel is for $Z=0.1 Z \odot$. Because the spin parameter of the $\mathrm{BHs}$ tends to decrease with the number of mergers, a correlation exists between mass and spin of the BHs, with the heavier BHs having the smaller spins.

Eq. (29) by evolving a synthetic population of $N_{\mathrm{NC}}=100$ cluster models with mass and radius distributed uniformly below the solid-red line in Fig. 9, and impose the conditions $M_{\mathrm{cl}} \leq 10^{8} M_{\odot}$, and $\rho_{\mathrm{h}, 0} \leq 10^{7} M_{\odot} \mathrm{pc}^{-3}$. We evolved these models until a time $t_{\mathrm{f}}=\left(2^{3 / 2}-1\right) \frac{2}{3} \tau_{\mathrm{rh}, 0} / \zeta$, after which time the clusters have expanded significantly and $r_{\mathrm{h}}\left(t_{\mathrm{f}}\right)=2 r_{\mathrm{h}, 0}$. The basic assumption is that there exists a constant number of clusters in the high density region of parameter space where hierarchical growth occurs - this requires replenishment of the clusters on a time-scale $\sim t_{\text {f }}$ (i.e., several times the cluster relaxation timescale). Under this assumption, the merger rate of large $\mathrm{BHs}$ for each simulated cluster is simply $\Gamma_{\mathrm{m}} \simeq N_{\mathrm{m}} / t_{\mathrm{f}}$, with $N_{\mathrm{m}}$ the number of BH binary mergers in the mass range $\mathcal{M}=\left[10^{2}, 10^{3}\right] M_{\odot}$. We evolved two sets of models with $Z=Z_{\odot}$ and $0.1 Z_{\odot}$, and found that they produce a $\mathrm{BH}$ merger rate per volume of $\Gamma_{\mathrm{V}} \simeq 0.04 f_{\mathrm{BH}} \mathrm{Gpc}^{-3} \mathrm{yr}^{-1}$ 
and $0.06 f_{\mathrm{BH}} \mathrm{Gpc}^{-3} \mathrm{yr}^{-1}$, respectively. This merger rate can be compared with existing limits derived from the nondetection of IMBHs by Advanced LIGO. For 100-20 $M_{\odot}$ binaries, Abbott et al. (2017b) reported an upper limit to the merger rate of $\lesssim 10 \mathrm{Gpc}^{-3} \mathrm{yr}^{-1}$ at $90 \%$ confidence, broadly consistent with our findings.

Finally, from our models we compute the detection rate of large BHs by Advanced LIGO/Virgo as

$\Gamma_{\mathrm{obs}} \simeq \frac{d N_{g x}}{d V} f_{\mathrm{BH}} f_{\mathrm{NC}} \frac{1}{N_{\mathrm{NC}}} \sum_{i=1}^{N_{\mathrm{NC}}} \frac{1}{t_{\mathrm{f}, i}} \sum_{j=1}^{N_{\mathrm{m}}} \frac{4 \pi}{3} D_{j}^{3}$

where $D_{j}$ is the luminosity distance out to which the ring-down phase of the $j^{\text {th }}$ event can be detected with a $\mathrm{S} / \mathrm{N}$ of at least 10 . We computed $D_{j}$ using Eq. (A17) in Flanagan \& Hughes (1998), and ignore cosmological corrections which for current interferometers are small (e.g., Miller 2002 ). For $Z=Z_{\odot}$, our models give a detection rate $\Gamma_{\mathrm{LIGO}} \simeq$ $0.3 f_{\mathrm{BH} \mathrm{yr}^{-1}}$ for events with total mass $>100 M_{\odot}$. Even a small contribution from lower metallicity clusters increases substantially these rates. Taking $Z=0.1 Z_{\odot}$, our models give $\Gamma_{\text {LIGO }} \simeq 3 f_{\mathrm{BH}} \mathrm{yr}^{-1}$. Thus, if $\sim 10 \%$ of the local high density/mass NCs have such metallicities (or lower), then the total detection rate will be increased to $\Gamma_{\text {LIGO }} \simeq 0.6 f_{\mathrm{BH}} \mathrm{yr}^{-1}$.

$\mathrm{BHs}$ that are formed from previous mergers admit a unique mass/spin correlation that could allow our model to be tested in the near future. This is demonstrated in Fig. 10 where we show the mass and spin distribution of the large $\mathrm{BHs}$ formed in the $\mathrm{NC}$ models. After the first few mergers, we expect $\chi \approx 0.7$ (Gerosa \& Berti 2017; Fishbach et al. 2017). But, as the random walk process of accretion of smaller BHs progresses, $\chi$ decreases so that the larger BHs will also have the smaller spins (e.g., Mandel et al. 2008). For $\mathcal{M} \gtrsim 200 M_{\odot}$, we find that spins are typically confined in the range $\lesssim 0.6$. The final spin distribution is sensitive to the initial mass function of the BHs, and therefore to metallicity. Because low metallcity stars produce larger BHs, fewer mergers are needed to reach a certain mass, resulting in a larger spin parameter at a given $\mathcal{M}$.

\subsection{Formation of massive black hole seeds}

The end-product of our merger scenario is the production of IMBHs. Subsequently to their formation, these seeds could then grow by swallowing stars and/or through standard Eddington-limited accretion of gas to become the massive BHs we observe today. A weak test to this idea is provided in Fig. 9. Here we show the population of NCs with strong evidence for a massive BH (purple symbols) and NCs with only an upper limit to the mass of a putative central $\mathrm{BH}$ (orange symbols). We find a sharp transition from clusters without to with evidence of a central $\mathrm{BH}$ at mass larger than several $10^{6} M_{\odot}$. This transition is consistent with our previous analysis showing that the formation of $\mathrm{BH}$ seeds should be favoured for clusters with masses larger than this value (see Section 3.1). For our scenario to be valid, however, we require not only that the cluster masses are large, but also that the clusters are initially sufficiently dense to allow for rapid growth. Hence, we compare the present-day clusters' radii to the radii of cluster models with $\rho_{\mathrm{h}, 0}=10^{5} M_{\odot} \mathrm{pc}^{-3}$ and $v_{\mathrm{esc}, 0}=300 \mathrm{~km} \mathrm{~s}^{-1}$ that we evolved using Eq. (4) for
1, 5 and $10 \mathrm{Gyr}$ (dashed-red lines). All, but one, of the observed systems have radii that are near, although somewhat larger than, the predicted radii after $10 \mathrm{Gyr}$ of evolution. The radii as a function of mass are similar to the results in Gieles et al. (2010), albeit somewhat larger. This is because we fixed $\psi=5$, where in reality the clusters at $10 \mathrm{Gyr}$ have narrower mass functions and hence $\psi \approx 2$. However, the initial radii could have been much smaller, because the Universe was much denser in the past, by a factor $(1+z)^{3}$. This suggests that significant expansion of the cluster, and $\mathrm{BH}$ growth, might have occurred in these systems over the Hubble time.

Finally, we note that episodic star formation and accretion of star clusters can lead to morphological and structural transformation of the nuclei which is difficult to address with our simplified models (e.g., Antonini 2013; Antonini et al. 2015). A self-consistent assessment of our scenario for massive $\mathrm{BH}$ seed formation will therefore require to model the cluster formation and evolution in a cosmological set. Binaries with masses between a hundred and a few thousand Solar masses have frequencies that will make them detectable by space-borne gravitational-wave observatories such as LISA (Amaro-Seoane et al. 2007; Amaro-Seoane 2018a). Third-generation detectors such as the proposed Einstein Telescope (Punturo et al. 2010) and the Cosmic Explorer (Abbott et al. 2017a) will be able to probe GWs in a frequency range reaching down to $\sim 1 \mathrm{~Hz}$ and detect $\mathrm{BH}$ mergers at high redshift. This will provide direct constraints on the proposed mechanism, which are independent of the uncertain assumptions about the dynamics and evolutionary history of the clusters.

FA acknowledges support from an STFC Rutherford fellowship (ST/P00492X/1) from the Science and Technology Facilities Council. MG acknowledges financial support from the Royal Society (University Research Fellowship) and the European Research Council (ERC-StG-335936, CLUSTERS).

\section{REFERENCES}

Abbott B. P., et al., 2016a, Physical Review Letters, 116, 061102 Abbott B. P., et al., 2016b, ApJ, 818, L22

Abbott B. P., et al., 2017a, Classical and Quantum Gravity, 34, 044001

Abbott B. P., et al., 2017b, Phys. Rev. D, 96, 022001

Abbott B. P., et al., 2017c, Physical Review Letters, 119, 141101

Alexander P. E. R., Gieles M., 2012, MNRAS, 422, 3415

Amaro-Seoane P., 2018a, Living Reviews in Relativity, 21, 4

Amaro-Seoane P., 2018b, Phys. Rev. D, 98, 063018

Amaro-Seoane P., Chen X., 2016, MNRAS, 458, 3075

Amaro-Seoane P., Gair J. R., Freitag M., Miller M. C., Mandel I., Cutler C. J., Babak S., 2007, Classical and Quantum Gravity, 24, R113

Anderson J., van der Marel R. P., 2010, ApJ, 710, 1032

Antonini F., 2013, ApJ, 763, 62

Antonini F., Perets H. B., 2012, ApJ, 757, 27

Antonini F., Rasio F. A., 2016, ApJ, 831, 187

Antonini F., Barausse E., Silk J., 2015, ApJ, 812, 72

Antonini F., Toonen S., Hamers A. S., 2017, ApJ, 841, 77

Antonini F., Rodriguez C. L., Petrovich C., Fischer C. L., 2018, MNRAS, 480, L58 
Askar A., Szkudlarek M., Gondek-Rosińska D., Giersz M., Bulik T., 2017, MNRAS, 464, L36

Banerjee S., Baumgardt H., Kroupa P., 2010, Monthly Notices of the Royal Astronomical Society, 402, 371

Barkat Z., Rakavy G., Sack N., 1967, Physical Review Letters, 18,379

Belczynski K., Kalogera V., Bulik T., 2002, ApJ, 572, 407

Belczynski K., Dominik M., Bulik T., O?Shaughnessy R., Fryer C., Holz D. E., 2010, The Astrophysical Journal, 715, L138

Belczynski K., Holz D. E., Bulik T., O?Shaughnessy R., 2016, Nature, 534, 512

Binney J., Tremaine S., 2011, No Title. Princeton University Press, http: //books.google.com/books?id=6mF4CKxlbLsC\&printsec

Böker T., Sarzi M., McLaughlin D. E., van der Marel R. P., Rix H.-W., Ho L. C., Shields J. C., 2004, AJ, 127, 105

Breen P. G., Heggie D. C., 2013, Monthly Notices of the Royal Astronomical Society, 432, 2779

Campanelli M., Lousto C., Zlochower Y., Merritt D., 2007, The Astrophysical Journal, 659, L5

Carollo C. M., Stiavelli M., de Zeeuw P. T., Mack J., 1997, AJ, 114,2366

Chen X., Amaro-Seoane P., 2017, ApJ, 842, L2

Chen K.-J., Woosley S., Heger A., Almgren A., Whalen D. J., 2014, ApJ, 792, 28

Choksi N., Volonteri M., Colpi M., Gnedin O. Y., Li H., 2018, preprint, (arXiv:1809.01164)

Christian P., Mocz P., Loeb A., 2018, ApJ, 858, L8

Conselice C. J., Blackburne J. A., Papovich C., 2005, ApJ, 620,564

Côté P., et al., 2006, ApJS, 165, 57

D'Orazio D. J., Samsing J., 2018, MNRAS, 481, 4775

Damour T., Gopakumar A., 2006, Phys. Rev. D, 73, 124006

Davies M. B., Miller M. C., Bellovary J. M., 2011, ApJ, 740, L42

Dominik M., Belczynski K., Fryer C., Holz D. E., Berti E., Bulik T., Mandel I., O'Shaughnessy R., 2013 , The Astrophysical Journal, 779, 72

Downing J. M. B., Benacquista M. J., Giersz M., Spurzem R., 2010, Monthly Notices of the Royal Astronomical Society, 407, 1946

Fishbach M., Holz D. E., Farr B., 2017, ApJ, 840, L24

Flanagan É. É., Hughes S. A., 1998, Phys. Rev. D, 57, 4535

Forbes D. A., Lasky P., Graham A. W., Spitler L., 2008, MNRAS, 389,1924

Fragione G., Ginsburg I., Kocsis B., 2018a, ApJ, 856, 92

Fragione G., Leigh N. W. C., Ginsburg I., Kocsis B., 2018b, ApJ, 867,119

Freitag M., Gürkan M. A., Rasio F. A., 2006, MNRAS, 368, 141

Fryer C. L., Kalogera V., 2001, The Astrophysical Journal, 554, 548

Georgiev I. Y., Böker T., 2014, MNRAS, 441, 3570

Georgiev I. Y., Hilker M., Puzia T. H., Goudfrooij P., Baumgardt H., 2009, MNRAS, 396, 1075

Georgiev I. Y., Böker T., Leigh N., Lützgendorf N., Neumayer N., 2016, MNRAS, 457, 2122

Gerosa D., Berti E., 2017, Phys. Rev. D, 95, 124046

Gerosa D., Berti E., O'Shaughnessy R., Belczynski K., Kesden M., Wysocki D., Gladysz W., 2018, preprint, (arXiv: 1808.02491)

Gieles M., Baumgardt H., Heggie D. C., Lamers H. J. G. L. M., 2010, MNRAS, 408, L16

Gieles M., Heggie D. C., Zhao H., 2011, MNRAS, 413, 2509

Gieles M., Balbinot E., Yaaqib R. I. S. M., Hénault-Brunet V., Zocchi A., Peuten M., Jonker P. G., 2018a, MNRAS, 473, 4832

Gieles M., et al., 2018b, MNRAS, 478, 2461

Giersz M., Leigh N., Hypki A., Lützgendorf N., Askar A., 2015,
MNRAS, 454, 3150

González J. A., Sperhake U., Brügmann B., Hannam M., Husa S., 2007a, Physical Review Letters, 98, 091101

González J. A., Hannam M., Sperhake U., Brügmann B., Husa S., 2007b, Physical Review Letters, 98, 231101

Goodman J., 1984, ApJ, 280, 298

Gültekin K., Miller M. C., Hamilton D. P., 2004, ApJ, 616, 221

Gültekin K., Miller M. C., Hamilton D. P., 2006, ApJ, 640, 156

Gürkan M. A., Freitag M., Rasio F. A., 2004, ApJ, 604, 632

Hamers A. S., Bar-Or B., Petrovich C., Antonini F., 2018, ApJ, 865,2

Heggie D., Hut P., 2003, The Gravitational Million-Body Problem: A Multidisciplinary Approach to Star Cluster Dynamics, by Douglas Heggie and Piet Hut. Cambridge University Press,

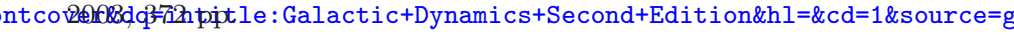

Hénon M., 1961, Annales d'Astrophysique, 24, 369

Hénon M., 1965, Annales d'Astrophysique, 28, 62

Hénon M., 1975, in Hayli A., ed., IAU Symposium Vol. 69, Dynamics of the Solar Systems. p. 133

Hobbs G., Lorimer D. R., Lyne A. G., Kramer M., 2005, Monthly Notices of the Royal Astronomical Society, 360, 974

Holley-Bockelmann K., Gültekin K., Shoemaker D., Yunes N., 2008, ApJ, 686, 829

Hurley J. R., Tout C. A., Pols O. R., 2002, Monthly Notices of the Royal Astronomical Society, 329, 897

Jeans J. H., 1919, MNRAS, 79, 408

Konstantinidis S., Amaro-Seoane P., Kokkotas K. D., 2013, A\&A, 557, A135

Kovetz E. D., Cholis I., Kamionkowski M., Silk J., 2018, Phys. Rev. D, 97, 123003

Kremer K., Chatterjee S., Rodriguez C. L., Rasio F. A., 2018, ApJ, 852, 29

Kroupa P., 2001

Kulkarni S. R., Hut P., McMillan S. J., 1993, Nature, 364, 421

Kupi G., Amaro-Seoane P., Spurzem R., 2006, MNRAS, 371, L45

Lanzoni B., et al., 2013, ApJ, 769, 107

Lee H. M., 1995, MNRAS, 272, 605

Liu B., Lai D., 2018, ApJ, 863, 68

Löckmann U., Baumgardt H., Kroupa P., 2010, MNRAS, 402, 519

Lousto C. O., Zlochower Y., 2008, Phys. Rev. D, 77, 044028

Lousto C. O., Zlochower Y., Dotti M., Volonteri M., 2012, Phys. Rev. D, 85, 084015

Lupi A., Colpi M., Devecchi B., Galanti G., Volonteri M., 2014, MNRAS, 442, 3616

MacLeod M., Trenti M., Ramirez-Ruiz E., 2016, ApJ, 819, 70

Mandel I., De Mink S. E., 2016, MNRAS, 458, 2634

Mandel I., Brown D. A., Gair J. R., Miller M. C., 2008, ApJ, 681,1431

Mapelli M., 2016, MNRAS, 459, 3432

Marchant P., Langer N., Podsiadlowski P., Tauris T. M., Moriya T. J., 2016, A\&A, 588

Miller M. C., 2002, ApJ, 581, 438

Miller M. C., Hamilton D. P., 2002a, MNRAS, 330, 232

Miller M. C., Hamilton D. P., 2002b, Monthly Notices of the Royal Astronomical Society, 330, 232

Miller M. C., Hamilton D. P., 2002c, ApJ, 576, 894

Miller M. C., Lauburg V. M., 2009, ApJ, 692, 917

Morscher M., Pattabiraman B., Rodriguez C., Rasio F. A., Umbreit S., 2015, The Astrophysical Journal, 800, 9

Nguyen D. D., et al., 2018, ApJ, 858, 118

O'Leary R. M., Kocsis B., Loeb A., 2009, Monthly Notices of the Royal Astronomical Society, 395, 2127

O'Leary R. M., Meiron Y., Kocsis B., 2016, ApJ, 824, L12

Peters P., 1964, Physical Review, 136, B1224

Petrovich C., Antonini F., 2017, ApJ, 846, 146

Plummer H. C., 1911, MNRAS, 71, 460 
Portegies Zwart S., McMillan S., 1999,

The Astrophysical Journal Letters, p. 12

Portegies Zwart S. F., McMillan S. L. W., 2002, ApJ, 576, 899

Portegies Zwart S. F., Dewi J., Maccarone T., 2004a, MNRAS, 355,413

Portegies Zwart S. F., Baumgardt H., Hut P., Makino J., McMillan S. L. W., 2004b, Nature, 428, 724

Punturo M., et al., 2010, Classical and Quantum Gravity, 27,084007

Quinlan G. D., 1996, NA, 1, 35

Quinlan G. D., Shapiro S. L., 1987, ApJ, 321, 199

Rezzolla L., Barausse E., Dorband E. N., Pollney D., Reisswig C., Seiler J., Husa S., 2008, Phys. Rev. D, 78, 044002

Rodriguez C. L., Antonini F., 2018, ApJ, 863, 7

Rodriguez C. L., Morscher M., Pattabiraman B., Chatterjee S., Haster C.-J., Rasio F. A., 2015, Physical Review Letters, 115, 051101

Rodriguez C. L., Chatterjee S., Rasio F. A., 2016, Physical Review D, 93, 084029

Rodriguez C. L., Amaro-Seoane P., Chatterjee S., Rasio F. A., 2018, Physical Review Letters, 120, 151101

Samsing J., 2017, preprint, (arXiv:1711.07452)

Samsing J., 2018, Phys. Rev. D, 97, 103014

Samsing J., D'Orazio D. J., 2018, MNRAS, 481, 5445

Samsing J., Askar A., Giersz M., 2018, ApJ, 855, 124

Seth A., Agüeros M., Lee D., Basu-Zych A., 2008, ApJ, 678, 116

Sigurdsson S., Hernquist L., 1993, Nature, 364, 423

Silsbee K., Tremaine S., 2017, ApJ, 836, 39

Spera M., Mapelli M., 2017, MNRAS, 470, 4739

Spera M., Mapelli M., Giacobbo N., Trani A. A., Bressan A., Costa G., 2018, preprint, (arXiv:1809.04605)

Spitzer Jr. L., Hart M. H., 1971, ApJ, 164, 399

Strader J., Chomiuk L., Maccarone T. J., Miller-Jones J. C. A., Seth A. C., 2012, Nature, 490, 71

Tanikawa A., 2013, MNRAS, 435, 1358

Tremou E., et al., 2018, ApJ, 862, 16

Turner M. L., Côté P., Ferrarese L., Jordán A., Blakeslee J. P., Mei S., Peng E. W., West M. J., 2012, ApJS, 203, 5

VanLandingham J. H., Miller M. C., Hamilton D. P., Richardson D. C., 2016, ApJ, 828, 77

Veitch J., Pürrer M., Mandel I., 2015, Physical Review Letters, 115,141101

Webb J. J., Leigh N. W. C., Singh A., Ford K. E. S., McKernan B., Bellovary J., 2018, MNRAS, 474, 3835

Woosley S. E., 2017, ApJ, 836, 244

Woosley S. E., Blinnikov S., Heger A., 2007, Nature, 450, 390

de Vita R., Trenti M., MacLeod M., 2018, MNRAS, 475, 1574 